\title{
New or poorly-known species of the millipede genus Tracbyjulus Peters, 1864 (Diplopoda: Spirostreptida: Cambalopsidae)
}

\author{
Новые и малоизвестные виды многоножкек рода Tracbyjulus \\ Peters, 1864 (Diplopoda: Spirostreptida: Cambalopsidae)
}

\section{S.I. Golovatch ${ }^{1}$, J.-J. Geoffroy ${ }^{2}$, J.-P. Mauriès ${ }^{3} \&$ D. VandenSpiegel ${ }^{4}$ С.И. Головач ${ }^{1}$, ЖК.-ЖК. ЖКоффруа ${ }^{2}$ ЖК.-П. Морьес ${ }^{3}$, А. ВанденШпигекь ${ }^{4}$}

\author{
${ }^{1}$ Institute for Problems of Ecology and Evolution, Russian Academy of Sciences, Leninsky pr. 33, Moscow 119071, Russia. \\ ${ }^{1}$ Институт проблем экологии и эволюции РАН, Ленинский пр. 33, Москва 119071 Россия. \\ ${ }^{2}$ Muséum national d'Histoire naturelle, Département Ecologie \& Gestion de la Biodiversité, UMR 7204 CERSP du CNRS, Equipe \\ EVOLTRAIT, 4, avenue du Petit Château, F-91800 Brunoy, France. \\ ${ }^{3}$ Muséum national d'Histoire naturelle, Département Systématique et Evolution, \\ Section Arthropodes, Case Postale n53, 61 rue Buffon F-75231 Paris Cedex 05, France. \\ ${ }^{4}$ Musée Royal de l'Afrique centrale, B-3080 Tervuren, Belgium.
}

KEY WORDS: Diplopoda, Trachyjulus, taxonomy, new species, cave, Thailand, Malaysia, Vietnam, Nepal.

КЛЮЧЕВЫЕ СЛОВА: Diplopoda, Trachyjulus, таксономия, новый вид, пещера, Китай, Таиланд, Малайзия, Вьетнам, Непал.

ABSTRACT. The large genus Trachyjulus, in which endemic species range from Sri Lanka and India in the west, through Indochina and the Himalaya, to Malay Peninsula and Indonesia (Sumatra and Java), in the east, currently encompasses 31 species, including four new: T. beroni sp.n. and T. subcalvus sp.n., both from Malaysia, as well as T. unciger sp.n. and T. phylloides sp.n., both from Thailand. In addition, brief redescriptions and abundant illustrations are provided for T. singularis (Attems, 1938) from southern Vietnam and several provinces of Thailand, as well as for $T$. tjampeanus (Attems, 1903) from Java, whereas the pantropical T. calvus (Pocock, 1893), based on fresh material from Nepal, is a senior subjective synonym of T. nordquisti (Attems, 1909) and T. nordquisti ambiguus Mauriès, 1983 (both syn.n.).

РЕЗЮМЕ. Большой род Trachyjulus, эндемичные виды которого распространены от Шри Ланки и Индии на западе, через Гималаи и Индокитай до Малайского полуострова и Индонезии (Суматра и Ява) на востоке, ныне насчитывает 31 вид, в т.ч. четыре новых: T. beroni sp.n. и T. subcalvus sp.n., оба из Малайзии, а также $T$. unciger sp.n. и $T$. phylloides sp.n., оба из Таиланда. Кроме того, кратко переописаны и подробно проиллюстрированы виды T. singularis (Attems, 1938) с юга Вьетнама и из ряда провинций Таиланда и T. tjampeanus (Attems, 1903 ) с Явы, а пантропичекий вид T. calvus (Pocock, $1893)$ по свежему материалу из Непала - старший субъективный синоним T. nordquisti (Attems, 1909) и T. nordquisti ambiguus Mauriès, 1983 (оба syn.n.).

\section{Introduction}

The millipede genus Trachyjulus Peters, 1864 has relatively recently been reviewed and shown to comprise 28 species or subspecies [Jeekel, 2004], all arranged below in alphabetical order:

1. T. aelleni Mauriès, 1981 - Sri Lanka, Central Province, Hanguranketa, Cave Instripura [Mauriès, 1981].

2. T. annectens Silversti, 1923 - India, Madras [Silvestri, 1923].

3. T. butteli (Carl, 1922) - Indonesia, Sumatra, Bandar Baroe [Carl, 1922, 1941]; originally described as Cambalopsis butteli. First transferred to Trachyjulus by Jeekel [1963].

4. T. calvus (Pocock, 1893) - Myanmar, Reef Island near Tavoy and Palon in Pegu [Pocock, 1893]; originally described as Cambala calva. Later recorded in Indonesia, Sumatra, Fort de Kock, as Cambalopsis calva in Borneo, Sarawak, Kuching and in India, Bengal, Calcutta [Silvestri, 1895; Pocock. 1894, 1895; Attems, 1914, 1936], as Cambala calva in Malay Peninsula, Kedah, Baling [Wang \& Tang, 1965]; redescribed from type material as Cambalopsis calvus [Mauriès, 1970], first transferred to Trachyjulus by Jeekel [1963].

5. T. cavernicola (Pocock, 1894) — Indonesia, Sumatra, Ngalau Cavern near Pajacombo [Pocock, 1894]; originally described as Cambala cavernicola. Later recorded also from Singapore, Bukit Timah [Wang, 1967]. First transferred to Trachyjulus by Jeekel [1963].

6. T. ceylanicus Peters, 1864 - the type species, originally described from Sri Lanka, Rambodde [Peters, 
1864], later recorded also from Sri Lanka, Pundel-Oya Valley, Paradenia and Trincomalie [Humbert, 1865], Punduloya and India, Madras [Pocock, 1892], as well as again from Sri Lanka, Sabaragamuwa Province, Nonpareil, 3 mi NE of Belihul-Oya [Mauriès, 1981].

7. T. costatus Verhoeff, 1936 - Sri Lanka, Demodera. Originally described as $T$. ceylonicus costatus (sic!) [Verhoeff, 1936], but later allotted the rank of a full species [Jeekel, 1963].

8. T. dentatus (Pocock, 1894) - Indonesia, Java, Batavia [Pocock, 1894); originally described as Cambala dentata. Later recorded also in Malaysia, Kedah, Baling [Wang \& Tang, 1965].

9. T. fissispinus (Attems, 1930) - Indonesia, Sumatra, Fort de Kock, Cave Pauh (Durian cave) [Attems, 1930]; originally described as Cambalopsis fissispina.

10. T. heteropus Silvestri, 1923 - Malay Peninsula, Patani, Jalor [Silvestri, 1923].

11. T. humberti Carl, 1911 - Sri Lanka, without precise locality [Carl, 1911].

12. T. lankanus Mauriès, 1981 — Sri Lanka, Sabaragamuwa Province, Deerwood, Kuruwita 6 mi NNW of Ratnapurna [Mauriès, 1981].

13. T. mimus Silvestri, 1924 - India, Assam, Garo Hills, Siju Cave [Silvestri, 1924].

14. T. minor Silvestri, 1923 — Sri Lanka, Namunakuli [Silvestri, 1923]; originally described as T. ceylanicus minor, but later allotted the rank of a full species [Jeekel, 1963].

15. T. modestior Silvestri, 1923 - India, Cochin State, Forest Tramway, miles 10-14 [Silvestri, 1923].

16. T. modiglianii (Silvestri, 1895) — Indonesia, Sumatra, Si-Rambe [Silvestri, 1895]. Originally described as Cambala modiglianii, later transferred to Trachyjulus by Jeekel [1963].

17. T. nordquisti Attems, 1909 - Singapore, Botanical Garden [Attems, 1909], later recorded also from Bismarck Archipelago, New Britain, Papua New Guinea; Matupi, in Malaysia; Moluccas, Ambon Island, Cave Liang ikan, and at Pare-Pare, Sulawesi, both in Indonesia; at Brava, Cape Verde Islands, as well as in several places in Brazil and Guiana [see Jeekel, 2004]. Hoffman [1977] was the first to unequivocally suspect $T$. nordquisti to actually represent a junior synonym of T. calvus.

18. T. nordquisti ambiguus Mauriès, 1983 - Thailand, Kanchanaburi Prov., Cave Daowdaeng [Mauriès, 1983].

19. T. pauper Silvestri, 1923 — India, Xchilkada [Silvestri, 1923].

20. T. proximatus Silvestri, 1923 - India, Barkuda, Lake Chilka [Silvestri, 1923].

21. T. rivicola (Attems, 1930) - Indonesia, Sumatra, Muara Klingi, Musi River [Attems, 1930].

22. T. silvestrii Hoffman, 1977 - Malaysia, Malay Peninsula, Selangor, $22 \mathrm{~km}$ from Kuala Lumpur, Cave Gua Anak Takun at Templar Park [Hoffman, 1977].

23. T. simulans (Carl, 1941) - India, Coonoor and Mudumalai, Gudalur [Carl, 1941]. Originally described as Cambalopsis simulans, later transferred to Trachyjulus by Jeekel [1963].

24. T. singularis (Attems, 1938) - Vietnam, Cochinchina, Poulo-Condore Island [Attems, 1938]. Originally described as Cambalopsis singularis, later transferred to Trachyjulus by Jeekel [1963].

25. T. tjampeanus (Attems, 1903) - Indonesia, Java, Cave Tjampea and Tjibodas [Attems, 1903; Chamberlin, 1945]. Originally described as Cambalopsis tjampeana, later transferred to Trachyjulus by Jeekel [1963].

26. T. willeyi (Carl, 1941) — Sri Lanka, numerous localities [Jeekel, 2004]. Originally described as Trachyjulus humberti var. willeyi [Carl, 1941], later allotted the rank of a full species by Jeekel [1963].

27. T. willeyi montanus Mauriès, 1981 - Sri Lanka, Sabaragamuwa Province, Kargal-Oya, 3 mi ENE of Belihul-Oya [Mauriès, 1981].

28. T. wilsonae Mauriès, 1983 - Nepal, several localities [Mauriès, 1981; Jeekel, 2004].

More information can be obtained from Jeekel's [2004] catalogue which is still fully relevant concerning Trachyjulus. Over half of the species have been keyed as well [Jeekel, 1963].

This genus can be characterized as follows:

\section{Trachyjulus Peters, 1864}

DIAGNOSIS. Ocelli few (up to 6), arranged in a vertical row. Mentum usually devoid of a transverse suture, remaining undivided into a promentum and a eumentum. Collum, sometimes also several following segments, smooth (= acarinate) or nearly so at least dorsally. Collum usually not particularly strongly inflated compared to postcollum constriction. Midbody metazona strongly carinate; longitudinal crests often nearly complete, almost undivided; the typical carinotaxy formula as follows: $10-3 / 10-3+\mathrm{I} / \mathrm{i}+2 /$ $2+\mathrm{m} / \mathrm{m}$; lateral crests growing increasingly poorly developed ventrad; axial crest $(\mathrm{m} / \mathrm{m})$ a little lower than adjacent ones.

$\sigma^{7}$ leg 1 strongly reduced to a broad transverse coxosternum showing a pair of central, often completely fused coxal processes flanked by 1-segmented, more or less rudimentary telopodites. $\sigma^{7}$ legs 2 nearly normal, only coxae evidently elongated. $\sigma^{7}$ legs 3 only very slightly reduced in size, coxae very evidently elongated, at least 2 times as long as broad.

Anterior gonopods consisting of a coxosternum supporting two, only basally fused/contiguous, diverging, subtriangular halves, each latter composed of a large, distolateral, basally often poorly delimited, distally setose, subcylindrical, elongate, 1-segmented telopodite tightly adjusted to and subtended by one or two coxal processes, one medial, the other lateral (rarely this latter can be absent). Posterior gonopods contiguous medially over most of their length, usually crowned with a clear-cut, lateral, setose field and a higher, medial, more or less complex flagellum (rarely absent).

Type species: T. ceylanicus Peters, 1864. 
So defined, discarding the sole pantropical T. nordquisti (see below), the genus shows a quite coherent distribution pattern: Sri Lanka, India, Himalaya, Indochina, Malaya, Sumatra and Java [Hoffman, 1977].

The present paper treats species of the genus Trachyjulus, thus continuing our efforts in putting on record the numerous and diverse Cambalopsidae collected mainly in caves in Southeast Asia during a few past decades [Golovatch et al., 2007a, b, 2009, 2011a, b, $\mathrm{c}, \mathrm{d}]$.

Abbreviations used:

MNHN - Muséum national d'Histoire naturelle, Paris, France;

MZB - Museum Zoologicum Bogoriense, Cibinong, Indonesia;

MZCU - Museum of Zoology, Chulalongkorn University, Bangkok, Thailand;

NMNHS - National Museum of Natural History, Sofia, Bulgaria;

VMNH - Virginia Museum of Natural History, Martinsville, Virginia, USA;

ZMUC - Natural History Museum, University of Copenhagen, Copenhagen, Denmark;

ZMUM - Zoological Museum, State University of Moscow, Moscow, Russia;

SEM — Scanning electron microscopy.

\section{Material and methods}

The material serving as the basis for the present contribution derives from the predominantly subterranean collections made in Indonesia, Thailand and Vietnam by Anne Bedos and/or Louis Deharveng (MNHN), sometimes together with their collaborators, in Indonesia also by Josiane Lips (France), in Malaysia by Petar Beron (NMNHS), in Malaysia and Thailand by Helmut Steiner (Germany), in Thailand also by Philippe Leclerc and Christine Dutin (both France), as well as by Somsak Panha and his collaborators from the Animal Systematics Research Unit, MZCU: Chirasak Sutcharit, Natdanai Likhitrakarn, Piyatida Pimvichai and some others (MZCU). The field trips of 2009 and 2010 across Thailand, organized by Somsak Panha, were also attended by the first author. The bulk of this material, including most of the holotypes, has been deposited in MNHN, with several paratypes shared between the collections of MZB, MZCU, ZMUM, ZMUC, NMNHS and $\mathrm{VMNH}$, as indicated hereafter.

SEM micrographs were taken using a JEOL JSM6480LV scanning electron microscope. After examination, SEM material was removed from stubs and returned to alcohol, all such samples being kept at MNHN.

In the catalogue sections, D stands for the original description, $\mathrm{N}$ for additional descriptive notes, and $\mathrm{R}$ for a mere mention or record. Our catalogue sections omit some of the most dubious or unreliable records, focusing instead on the main literature alone.
Given only a few new or known species yielded, no key has been attempted here. However, diagnoses of all encountered species are provided.

\section{Taxonomic part}

Trachyjulus singularis (Attems, 1938) Figs 1-5.

Cambalopsis singularis Attems, 1938: 273 (D)

Trachyjulus singularis — Jeekel, 1963: 152, 154 (N); Jeekel, 2004: 49 (R)

MATERIAL. Type series (syntypes) of Cambalopsis singularis, $21 \sigma^{\top} \sigma^{7}, 21$ 우, 7 juv. (MNHN GA 021+micropreparation), Cochinchina (= southern Vietnam), Poulo-Condore Island, littoral, 04.1931, C. Dawydoff.

OTHER MATERIAL. $1 \sigma^{7}, 1$ (MNHN GA 093), Vietnam, Kien Giang Prov., Kien Luong Distr., Hon Chong, Nui Hang Tien, Hang Tien, cave, 8.11.1993, leg. L. Deharveng, Truong Quang Tam \& Duong Tien Dung (VIET-012); $1 \sigma^{7}$ (MNHN GA 093), $1 \sigma^{7}$ (SEM), same locality, Hang Tien, cave, 26.01.2003, leg. L. Deharveng \& A. Bedos (VIET-915); 1 ○ (MNHN GA 093), 1 우 (SEM), same locality, Hon Chong, Nui Bai Voi, hospital cave "grotte des Vaches de Bai Voi", 29.01.2003, leg. L. Deharveng \& Le Kong Man (VIET-938); $2 \sigma^{7} \sigma^{\top}, 2$ 우 (MNHN GA 093), 1 ○ (SEM), same locality, hospital-cave of Mo So, 29.01.2003, leg. L. Deharveng \& A. Bedos (VIET-943); $6 \sigma^{7} \sigma^{7}, 10$ 90, 2 juv. (MNHN GA 093), same locality, Cave 1 south of Bai Voi Pass, 2.12.2005, leg. L. Deharveng \& A. Bedos (Vn05-067); $2 \sigma^{7} \sigma^{\top}, 2$ 우 (MNHN GA 093), $1 \sigma^{7}$ (SEM), same locality, 2.12.2005, leg. L. Deharveng

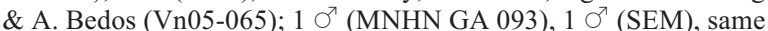
locality, Nui Bai Voi, cirque du Français, cave, 23.08.2003, leg. L. Deharveng \& A. Bedos (Vn0308-105); 1 \% , 3 oᄋ (MNHN GA 093), 1 O (SEM), same locality, Hon Chong, northern Nui Khoe La, cave, berleseate, 9.10.2005, leg. Dang Quoc Quan (Vn05-001); $2 \sigma^{7} \sigma^{7}, 2$ o+ 1 fragm. (MNHN GA 093), $1 \sigma^{7}$ (SEM), same locality, Nui Khoe La, South Cave of Khoe La, 4.12.2005, leg. L. Deharveng \& A. Bedos (Vn05-090); $2 \sigma^{7}, 3$ 우 (MNHN GA 093), 1 O (SEM), same locality, 6.06.2008, leg. L. Deharveng \& A. Bedos (Vn08-087); $30 \sigma^{7} \sigma^{7}, 32$ 우, 9 juv. (MNHN GA 093), 2

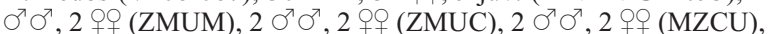
2 ○’ $^{\prime}, 2$ of (NMNHS), Thailand, Rayong Prov., Khao Cha Mao Distr., Wat Kao Loy, grotto, $13^{\circ} 03^{\prime} 27^{\prime \prime N}, 101^{\circ} 36^{\prime} 27^{\prime \prime E}, 60 \mathrm{~m}$, 23.10.2010, leg. S. Panha \& N. Likhitrakarn (No. 37); $6 \sigma^{7} \sigma^{7}, 11$ o+, 4 juv. (MNHN GA 093), $1 \sigma^{\top}, 1$ (ZMUM), 1 ○', 1 (ZMUC), 1 \%, 1 (MZCU), 1 \%, 1 क (NMNHS), Thailand, Chantaburi Prov., Kiang Hang Mau Distr., Kao Wong National Park, Cave Tham Kao Wong, 12 $53^{\prime} 52^{\prime \prime N}, 101^{\circ} 49^{\prime} 01^{\prime \prime E}, 50$ m, 24.10.2010, leg. S. Panha \& N. Likhitrakarn (No. 38); 1 \%, 3 oo, 1 fragm. (MNHN GA 093), Thailand, Chonburi Prov., Botong Distr., Wat Cha An Ont, Cave Tham Kao Cha An Ont, 1311'58"N, 10134'34"E, $20 \mathrm{~m}, 25.10 .2010$, leg. S. Panha \& N. Likhitrakarn

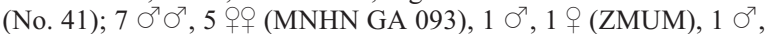

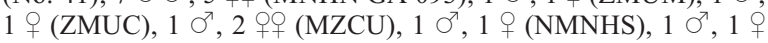
(VMNH), Thailand, Chonburi Prov., Botong Distr., Wat Kae Hayot, Cave Tham Kao Hayot, 1309'47"N, 101³5'52"E, 90 m, 25.10. 2010, leg. S. Panha \& N. Likhitrakarn (No. 42).

DIAGNOSIS. This species is easily distinguished from congeners in showing only one, medial, process of the anterior gonopod coxa, as well as in a simple, rather short, setiform flagellum of rather strongly elongated posterior gonopods.

DESCRIPTIVE NOTES. Length of adults 13-23 $\left(O^{7}\right)$ or $18-30 \mathrm{~mm}(+)$, width of midbody segments $0.8-1.0\left(\sigma^{7}\right)$ or $1.0-1.1 \mathrm{~mm}(+)$. Adult body with $37 \mathrm{p}-$ $59+6-2 \mathrm{a}+\mathrm{T}\left(\mathrm{O}^{7}\right)$ or $42-66 \mathrm{p}+2-4 \mathrm{a}+\mathrm{T}(+)$ segments, up to $73+\mathrm{T}$ [Attems, 1938]. Coloration in adults greybrown to dark castaneous brown, only rarely rather 

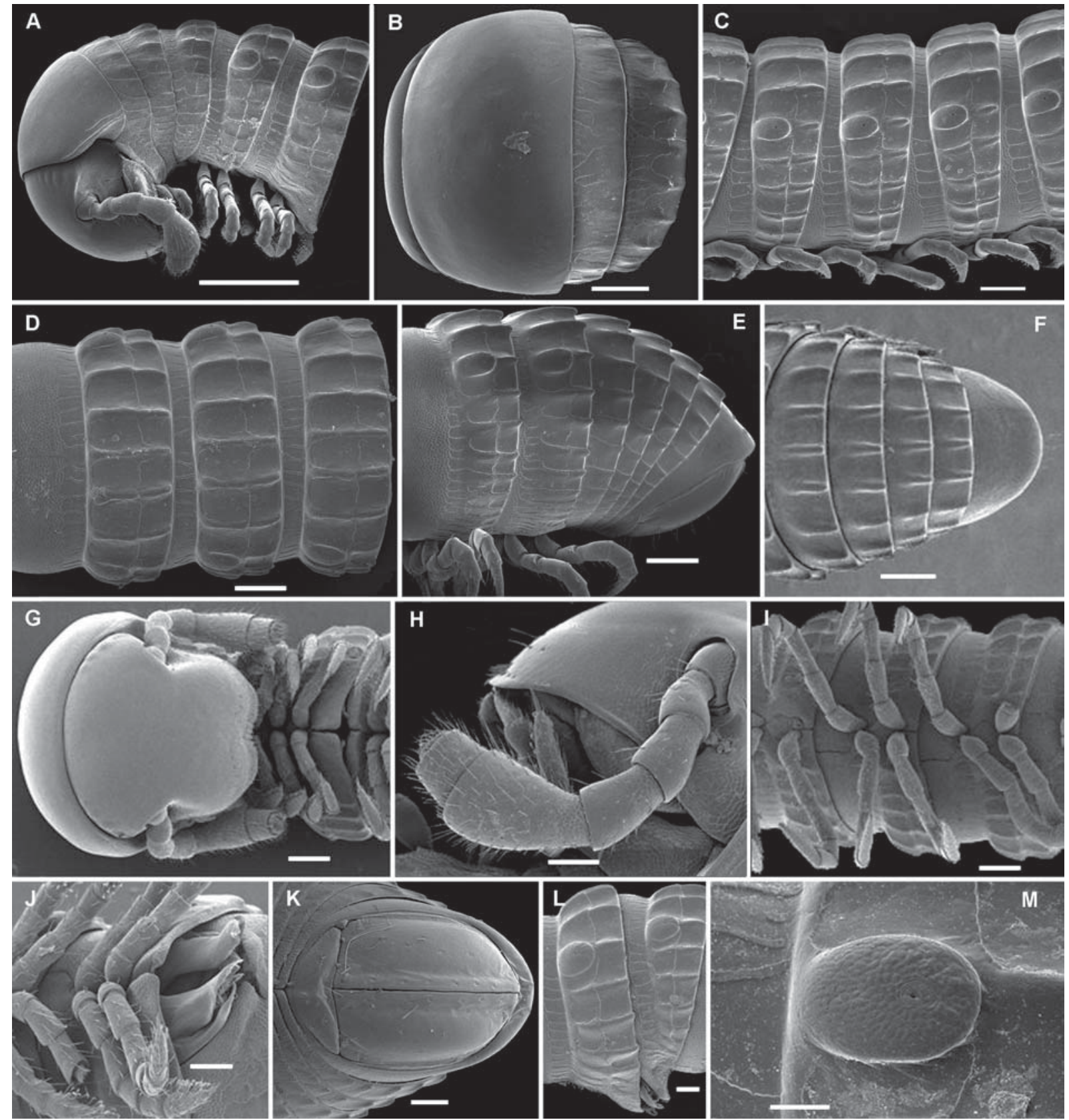

Fig. 1. Trachyjulus singularis (Attems, 1938), O $0^{7} 0^{7}$ from Hang Tien (VIET-915) (A-E \& K), northern Nui Khoe La (Vn05-001) (F), near South Cave (Vn05-090) (G \& I), inside South Cave (Vn05-090) (H \& L), Cave Mo So (VIET-943) (J) and inside and near Cave 1 of Mo So (Vn05-065) (M). A, B \& G - anterior body portion, lateral, dorsal and ventral views, respectively; C, D \& I — midbody segments, lateral, dorsal and ventral views, respectively; E, F \& K - posterior body portion, lateral, dorsal and ventral views, respectively; H antenna, lateral view; J \& L - segment 7, ventral and lateral views, respectively. Scale bars: 0.5 (A), 0.2 (B-G \& I), 0.1 (H \& J-L) \& 0.05 $\mathrm{mm}(\mathrm{M})$.

Рис. 1. Trachyjulus singularis (Attems, 1938), О О О из Hang Tien (VIET-915) (A-E, K), northern Nui Khoe La (Vn05-001) (F), близ пещеры South Cave (Vn05-090) (G и I), внутри пещеры South Cave (Vn05-090) (H, L), пещеры Mo So (VIET-943) (J) и внутри и близ пещеры Cave 1 of Mo So (Vn05-065) (M). A, B, G - передняя часть тела, соответственно сбоку, сверху и снизу; C, D, I среднетуловищные сегменты, соответственно сбоку, сверху и снизу; Е, F, K - задняя часть тела, соответственно сбоку, сверху и снизу; Н - антенна, сбоку; J, L - сегмент 7, соответственно снизу и сбоку. Масштаб: 0,5 (A), 0,2 (B-G, I), 0,1 (H, J-L), 0,05 мм (M).

uniformly dark blackish brown; pattern mostly variegated. Ocelli, metatergal crests and ozoporiferous tubercles blackish, a lateral stripe level to ozopores and one across vertex infuscate, black-brown. Clypeolabral region, collum and following $2-3$ segments, as well as antennae, spaces between metatergal crests, pleurosternal regions and basal podomeres usually lighter, yellowish brown. 

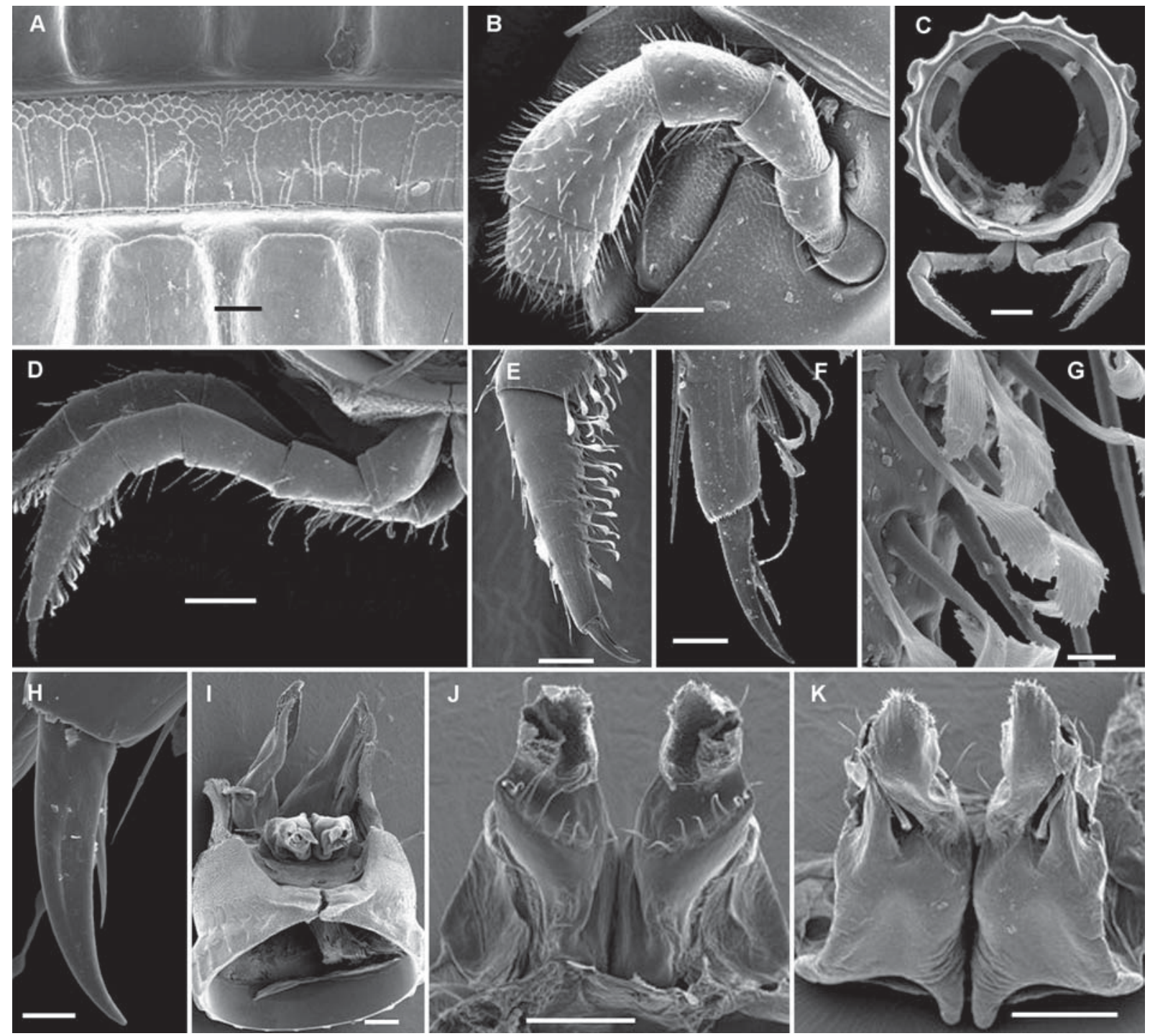

Fig. 2. Trachyjulus singularis (Attems, 1938), $0^{7} \mathrm{O}^{7}$ from northern Nui Khoe La (Vn05-001) (A \& E), "grotte des Vaches de Mo So" (VIET-938) (B), inside South Cave (Vn05-090) (C, G, J \& K), Cave Mo So (VIET-943) (D, F \& I) and Hang Tien (VIET-915) (H). A fine structure of tegument around stricture between pro- and metazona, dorsal view; B - antenna and gena, lateral view; C - crosssection of a midbody segment, caudal view; D — midbody legs, caudal view; E — tarsus and claw of a midbody leg, caudal view; F claw and end part of tarsus, caudal view; $\mathrm{G}$ - phylloid setae on tarsus, caudal view; $\mathrm{H}$ - claw, caudal view; I — segment 7 with gonopod block in situ, subventral view; J \& K - posterior gonopods, front and caudal views, respectively. Scale bars: 0.2 (C), 0.1 (B, D, I-K), 0.05 (E), $0.02(\mathrm{~A} \& \mathrm{~F}) \& 0.01 \mathrm{~mm}(\mathrm{G} \mathrm{\&} \mathrm{H})$.

Рис. 2. Trachyjulus singularis (Attems, 1938), О О $0^{7}$ из northern Nui Khoe La (Vn05-001) (А и E), пещеры "grotte des Vaches de Mo So" (VIET-938) (B), внутри пещеры South Cave (Vn05-090) (C, G, J, K), пещеры Mo So (VIET-943) (D, F, I) и Hang Tien (VIET-915) (H). А - тонкая структура покровов тела близ шва между про- и метазонитами, сверху; В - антенна и щека, сбоку; С поперечный разрез через среднетуловищный сегмент, сзади; D - среднетуловищные ноги, сзади; Е — лапка и коготок среднетуловищной ноги, сзади; F — коготок и дистальная часть лапки, сзади; G - листовидные щетинки на лапке, сзади; Н — коготок, сзади; I - сегмент 7 с гоноподным блоком на месте, почти снизу; J, K - задние гоноподы, соответственно спереди и снизу. Масштаб: 0,2 (C), 0,1 (B, D, I-K), 0,05 (E), 0,02 (A, F), 0,01 мм (G, H).

Usually $3+3$ ocelli in smaller individuals, $4+4$ or even $5+5$ in larger ones, always arranged into a single vertical row. Antennae short and stout (Figs 1A, G, H, 4A, 5A). Gnathochilarium (Figs 4B, 5B) moderately densely setose, mentum divided. Collum (Figs 1A, B \& G) smooth, only near lateral edge with 1-3 light, short, superficial striae. Segment 2 very faintly cristate, following metaterga with fully developed carinae (Figs $1 \mathrm{~A}-\mathrm{F}, \mathrm{L} \& \mathrm{M}, 2 \mathrm{~A} \& \mathrm{C}$ ), carinotaxy pattern of midbody segments typically 9-7/9$7+\mathrm{I} / \mathrm{i}+2 / 2+\mathrm{m} / \mathrm{m}$. Postcollum constriction evident, but not particularly strong (Fig. 1A). Telson bare and smooth, paraprocts densely setose (Fig. 1E, F \& K).

Legs short, nearly as long as midbody height (Figs 1A, C, E, I \& G, 2C, D). Ventral sides of $\sigma^{7}$ tibiae and tarsi with rows of peculiar, strong, distally flattened, phylloid to plumose setae (Figs 2D-G, 5F). A long 

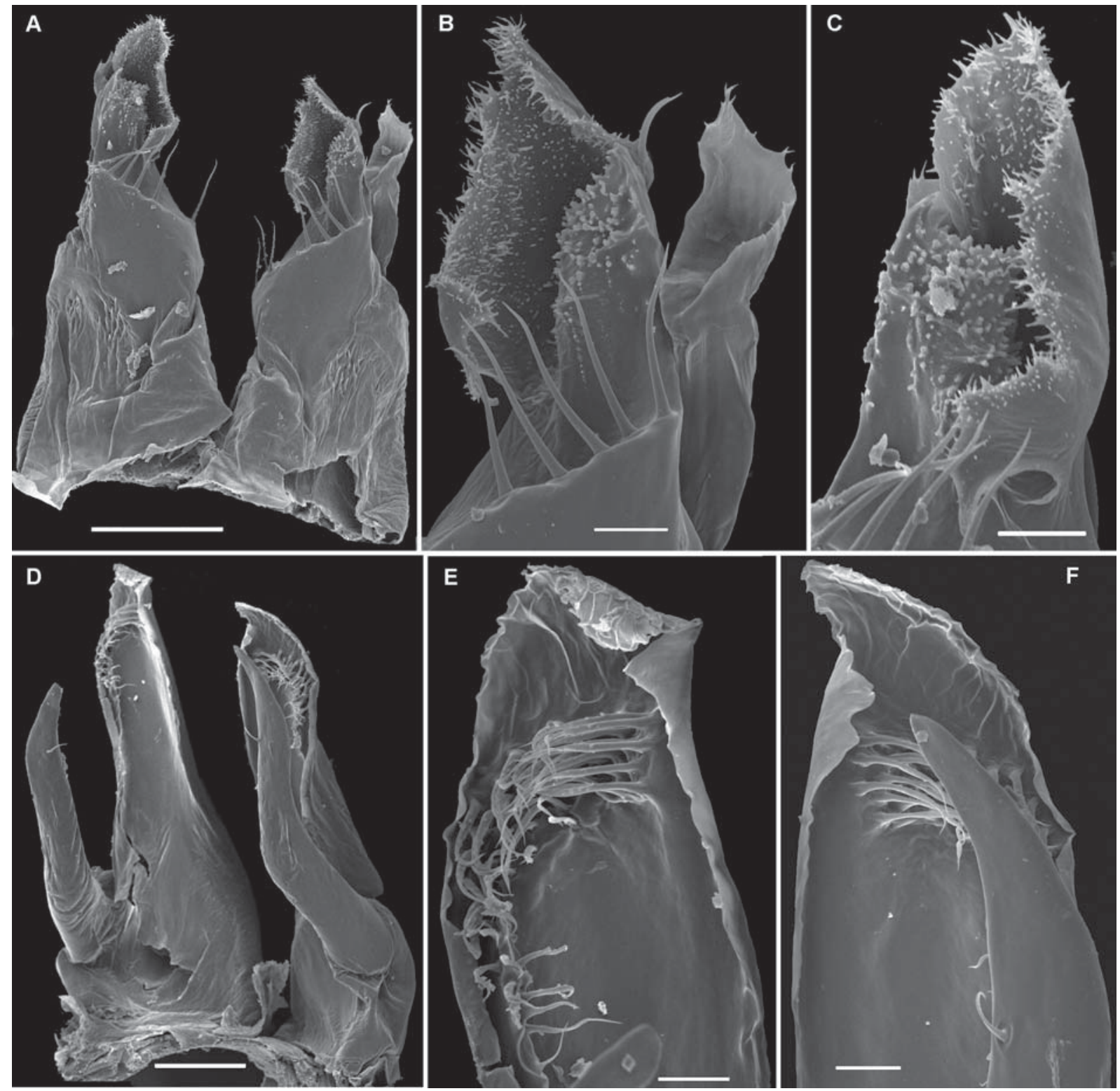

Fig. 3. Trachyjulus singularis (Attems, 1938), $\mathrm{O}^{7}$ from Hang Tien (VIET-915). A - posterior gonopods, front view; B \& C enlarged tip of posterior gonopods, front view; D - anterior gonopods, caudal view; E \& F — enlarged tip of anterior gonopods, caudal view. Scale bars: 0.1 (A \& D) \& $0.2 \mathrm{~mm}$ (B, C, E \& F).

Pис. 3. Trachyjulus singularis (Attems, 1938), О7 из Hang Tien (VIET-915). А - задние гоноподы, спереди; В, С - увеличенная вершина задних гоноподов, спереди; D - передние гоноподы, сзади; Е, F - увеличенная вершина передних гоноподов, сзади. Масштаб: 0,1 (A, D), 0,2 мм (B, C, E, F).

filament/spine at base of claw up to ca $2 / 3 \mathrm{rds}$ the length of claw itself (Fig. $2 \mathrm{~F} \& \mathrm{H}$ ).

$\mathrm{O}^{7}$ legs $1-3$ (Figs 4C, D, 5C-E) same as in numerous species of the Glyphiulus javanicus-group [Golovatch et al., 2007b, 2011c]; legs 1 with a subanchoriform, short, doubled, central process flanked by similarly short, setose, sac-shaped telopodites.

Anterior gonopods (Figs 2I, 3D-F, 4E, F, 5G) peculiar in lateral coxal process being missing, telopodite (te) very long and slender, nearly as long as an apically narrowed, setose and micrspiculate medial coxal pro- cess (mp). Posterior gonopods (Figs $2 \mathrm{I}-\mathrm{K}, 3 \mathrm{~A}-\mathrm{C}, 4 \mathrm{H}$, $\mathrm{G}, 5 \mathrm{H}$ ) short, stout, distally microserrate/spiculate, ending in a sharp hook, carrying a long setiform flagellum (fl) caudally and a row of strong setae frontally at about midway.

REMARKS. This species appears to be remarkably widespread in Indochina, having been found in various habitats (littoral, forest litter, grottos, caves) in Vietnam and Thailand (Map). At least in some of the localities, including the littoral of a remote isle off the coast of Vietnam, it seems to be anthropochore and/or pioneer. 


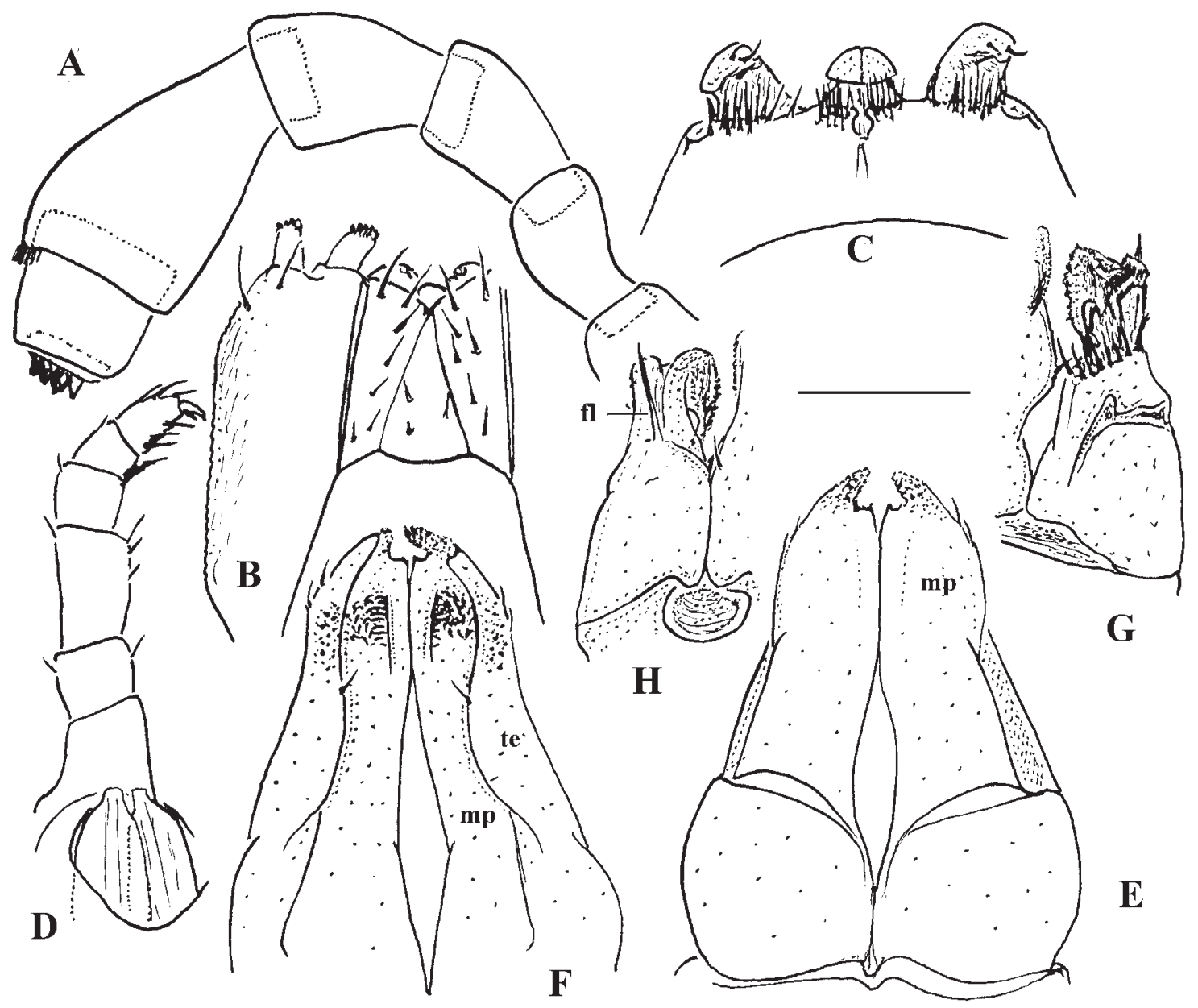

Fig. 4. Trachyjulus singularis (Attems, 1938), $\mathrm{O}^{7}$ from Hang Tien (VIET-915). A — antenna, lateral view; B — gnathochilarium, ventral view; C - legs 1, front view; D — leg 2, caudal view; E \& F — anterior gonopods, front and caudal views, respectively; G \& H left posterior gonopod, front and caudal views, respectively. Scale bar: $0.2 \mathrm{~mm}$.

Рис. 4. Trachyjulus singularis (Attems, 1938), О’ из Hang Tien (VIET-915). А — антенна, сбоку; В — гнатохилярий, снизу; С ноги 1, спереди; D - нога 2, сзади; Е, F — передние гоноподы, соответственно спереди и сзади; G, H — левый задний гонопод, соответственно спереди и сзади. Масштаб: 0,2 мм.

Trachyjulus tjampeanus (Attems, 1903) Figs 6 \& 7.

Cambalopsis tjampeana Attems, 1903: 68 (D)

Cambalopsis tjampeana — Attems, 1907: $130(\mathrm{~N})$; Attems 1914: 295 (R); Attems, 1926: 207 (N); Chamberlin, 1945: 27 (R)

Cambalopsis tyompeana — Attems, 1909: $74(\mathrm{~N})$

Trachyjulus tjampeanus - Jeekel, 1963: 152 (N); Jeekel, 2004: 49 (R)

MATERIAL. $1{ }^{7}, 1$ juv. (MNHN GA 94), Indonesia, Java, Tlogosari (Kebumen), Cave Goa Nglumprit, 16.08.2003, leg. J. Lips (No. 1309); 1 ○' (MNHN GA 94), 1 ○ (ZMUC), Java, Petruk (Kebumen), Cave Goa Petruk, 10.08.2003, leg. J. Lips (No. 1268); 1 9, 4 juv. (MNHN GA 94), Java, Petruk (Kebumen), Cave Goa Liah 3, 9.08.2003, leg. J. Lips (No. 1262); 1 ○' (ZMUM), Java, Jati Jajar (Kebumen), 19.08.2003, leg. J. Lips (No. 1357); 2 O+ (MNHN GA 94), Java, Tlogosari (Kebumen), Cave Goa Duren Renteng, 22.08.2003, leg. J. Lips (No. 1364); $2 \sigma^{\top} \sigma^{\top}, 1$ ㅇ (MNHN GA 94), 1 $\sigma^{7}$ (VMNH), Java, Tlogosari (Kebumen), Cave Goa Jeruk, 16.08.2003, leg. J. Lips (No. 1322); $1 \sigma^{\top}, 1$ q, 2 juv. (MZB), 1 (SEM), Java, Tlogosari (Kebumen), Cave Goa Macan, 15.08.2003, leg. J. Lips (No. 1292); $1 \sigma^{7}$ (NMNHS), Java, Redi Sari (Kebumen), Cave Goa Maling, 18.08.2003, leg. J. Lips (No. 1253); 2 우, 2 juv. (MZB), Java, Petruk (Kebumen), Cave Goa Liah, 7.08.2003, leg. J. Lips (No. 1239); 1 $\sigma^{7}, 5$ 우 (MZB), 1 ○ , 1 ㅇ (MNHN GA 94), Java, Jawa Tengah, Nusakambangan Isle, Cilacap, Cave Goa Ratu, 16.02.2011, leg. L. Deharveng, A. Bedos, Dito \& Rangga (JAVA-

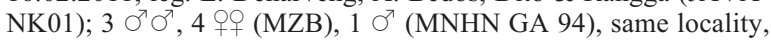
Cilacap S., Cave Goa Semen, 18.02.2011, leg. L. Deharveng, A. Bedos, Dito \& Sofiyan (JAVA-NK21); 1 ○', 3 우 (MZB), same locality, Cilacap S., Cave Goa Nagaraja, 19.02.2011, leg. L, Deharveng, A. Bedos, Dito \& Sofiyan (JAVA-NK26); 5 우 (MZB), Jawa Tengah, Nusakambangan Isle, Ujung Galang, Klaces, Cave Goa Maria, 22.02.2011, leg. L. Deharveng, A. Bedos, Dito \& Sofiyan (JAVA-NK51); 1 O (MZB), 1 ○ (MNHN GA 94), Jawa Timur, Punung, Cave Gua 4 of Kiuut, 26.07.2005, leg. L. Deharveng \& A. Bedos (JAVA-047); $1 \sigma^{7}, 1$ ( $(\mathrm{MZB}), 1 \sigma^{7}$ (MNHN GA 94), Jawa Timur, Tulakan, Bungur, Cave Gua Somopuro, 27.07.2005, leg. L. Deharveng \& A. Bedos (JAVA-060)

DIAGNOSIS. This species is easily distinguished from congeners in showing the medial coxal process of the anterior gonopod much longer than the lateral one, 


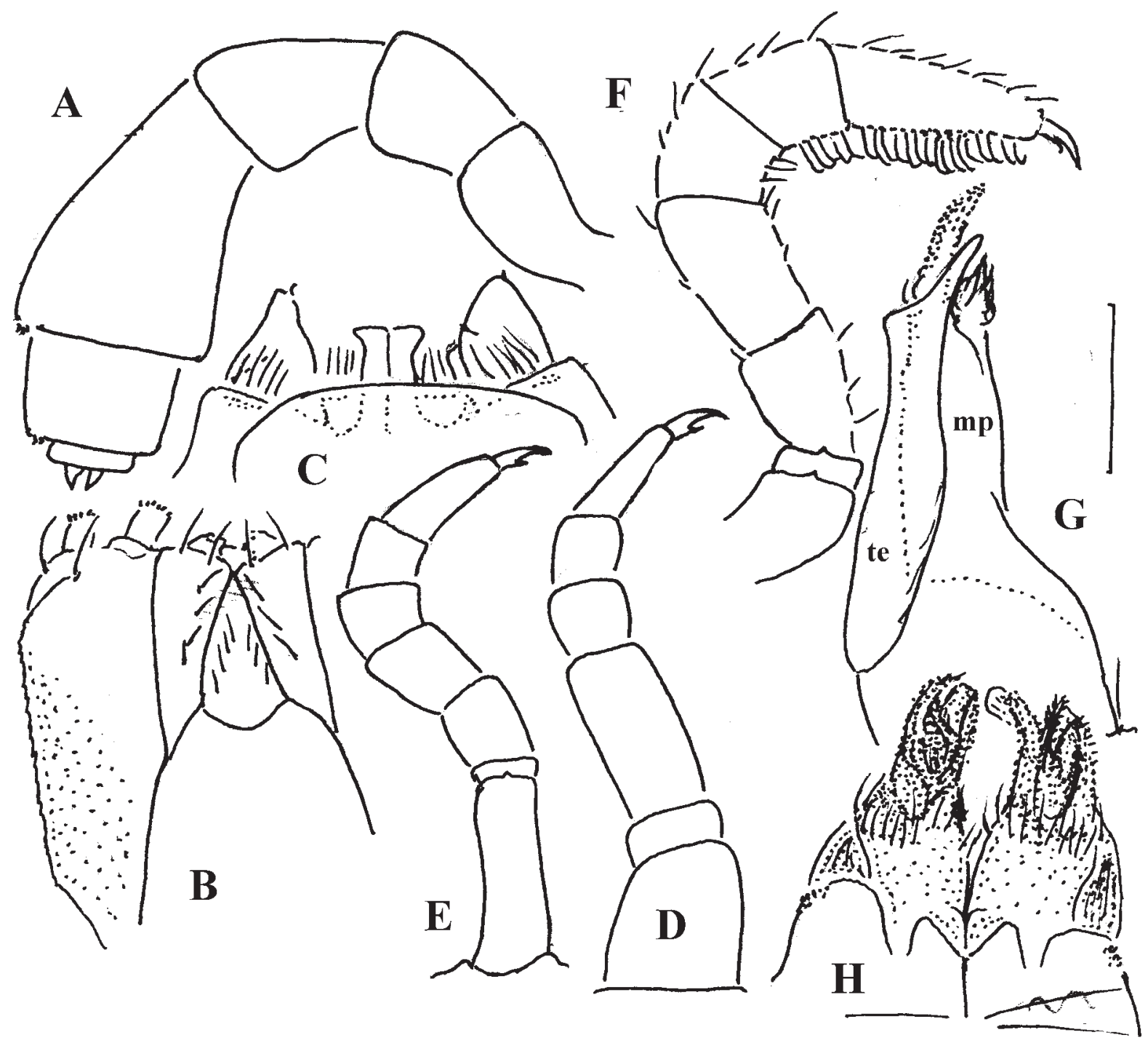

Fig. 5. Trachyjulus singularis (Attems, 1938), o from Cave Tham Kao Cha An Ont (No. 41). A — antenna, lateral view; B gnathochilarium, ventral view; C - legs 1, caudal view; D - leg 2, caudal view; E - leg 3, caudal view; F — midbody leg, caudal view; $\mathrm{G}$ - right anterior gonopod, caudal view; $\mathrm{H}$ - posterior gonopods, front view. Scale bar: $0.2 \mathrm{~mm}$.

Рис. 5. Trachyjulus singularis (Attems, 1938), От из пещеры Tham Као Cha An Ont (No. 41). А - антенна, сбоку; В гнатохилярий, снизу; C - ноги 1, сзади; D - нога 2, сзади; Е - нога 3, сзади; F — среднетуловищная нога, сзади; G - правый передний гонопод, сзади; Н - задние гоноподы, спереди. Масштаб: 0,2 мм.

as well as a deeply 3-branched flagellum of the posterior gonopod. It is widespread across Java (Map).

DESCRIPTIVE NOTES. Length of adults 9-23 $\mathrm{mm}\left(\sigma^{7},+\right)$, up to $30 \mathrm{~mm}$ in $\sigma^{7}$ and $37 \mathrm{in} \%$ [Attems, 1903]; width of midbody segments $0.7-1.1\left(\mathrm{O}^{7}\right.$, , $)$, up to 1.25 and $1.75 \mathrm{~mm}$ in $\sigma^{7}$ and $P$, respectively [Attems, 1903]. Adult body with $30 \mathrm{p}-59+4-2 \mathrm{a}+\mathrm{T}\left(\mathrm{O}^{1},+\right.$, $)$, up to $64+\mathrm{T}$ or $71+\mathrm{T}$ segments in $\sigma^{7}$ and $O$, respectively [Attems, 1903]. Coloration in adults rather uniformly light grey-brown to dark castaneous brown, without a clear-cut pattern. Ocelli brown to blackish.

$3+3$ ocelli in a single vertical row, obviously regardless of sex or body size. Antennae short and stout (Figs 6A, 7A). Gnathochilarium (Figs 7B) oligotrichous, mentum single. Collum (Figs 6A \& D) smooth, only near lateral edge with 1-3 light, short, superficial striae. Segment 2 evidently more poorly cristate compared to following metaterga, carinotaxy pattern of midbody segments typically 9-6/9$6+\mathrm{I} / \mathrm{i}+2 / 2+\mathrm{m} / \mathrm{m}$ (Fig. 6A-F). Postcollum constriction evident, but not particularly strong (Fig. 6A \& D). Telson bare and smooth, paraprocts densely setose (Fig. 6C \& E).

Legs short, a little less than midbody diameter (Fig. $6 \mathrm{~B}, \mathrm{C}, \mathrm{E}, \mathrm{F}, 7 \mathrm{~F})$. A long filament/spine at base of claw up to ca $2 / 3$ rds the length of claw itself (Figs 6G, 7F).

$\mathrm{O}^{7}$ legs 1-3 (Figs 7C-E) same as in numerous species of the Glyphiulus javanicus-group [Golovatch et al., 2007b, 2011c]; legs 1 (Fig. 7C) with a subanchoriform, rather short, doubled, central process flanked by 

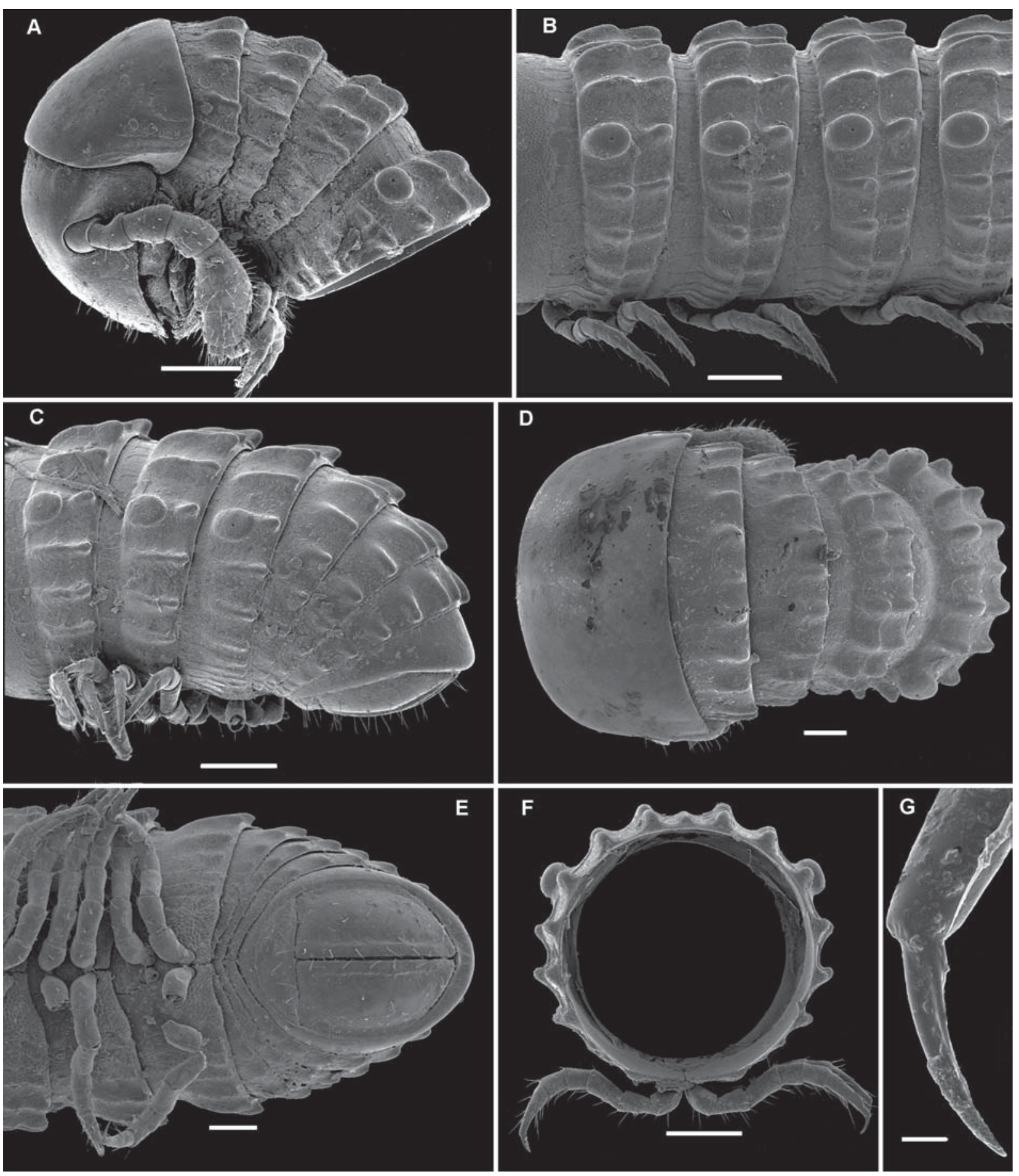

Fig. 6. Trachyjulus tjampeanus (Attems, 1903), o from Cave Goa Macan (No. 1292). A \& D - anterior body portion, lateral and dorsal views, respectively; B - midbody segments, lateral view; C \& E - posterior body portion, lateral and ventral views, respectively; F - cross-section of a midbody segment, caudal view; G - claw, caudal view. Scale bars: 0.2 (A-C \& F), 0.1 (D \& E) \& $0.01 \mathrm{~mm}(\mathrm{G})$.

Рис. 6. Trachyjulus tjampeanus (Attems, 1903),, из пещеры Goa Macan (No. 1292). A, D — передняя часть тела, соответственно сбоку и сверху; В - среднетуловищные сегменты, сбоку; $\mathrm{C}, \mathrm{E}$ - задняя часть тела, соответственно сбоку и снизу; $\mathrm{F}$ поперечный разрез через среднетуловищный сегмент, сзади; G - коготок, сзади. Масштаб: 0,2 (A-C, F), 0,1 (D, E), 0,01 мм (G). 


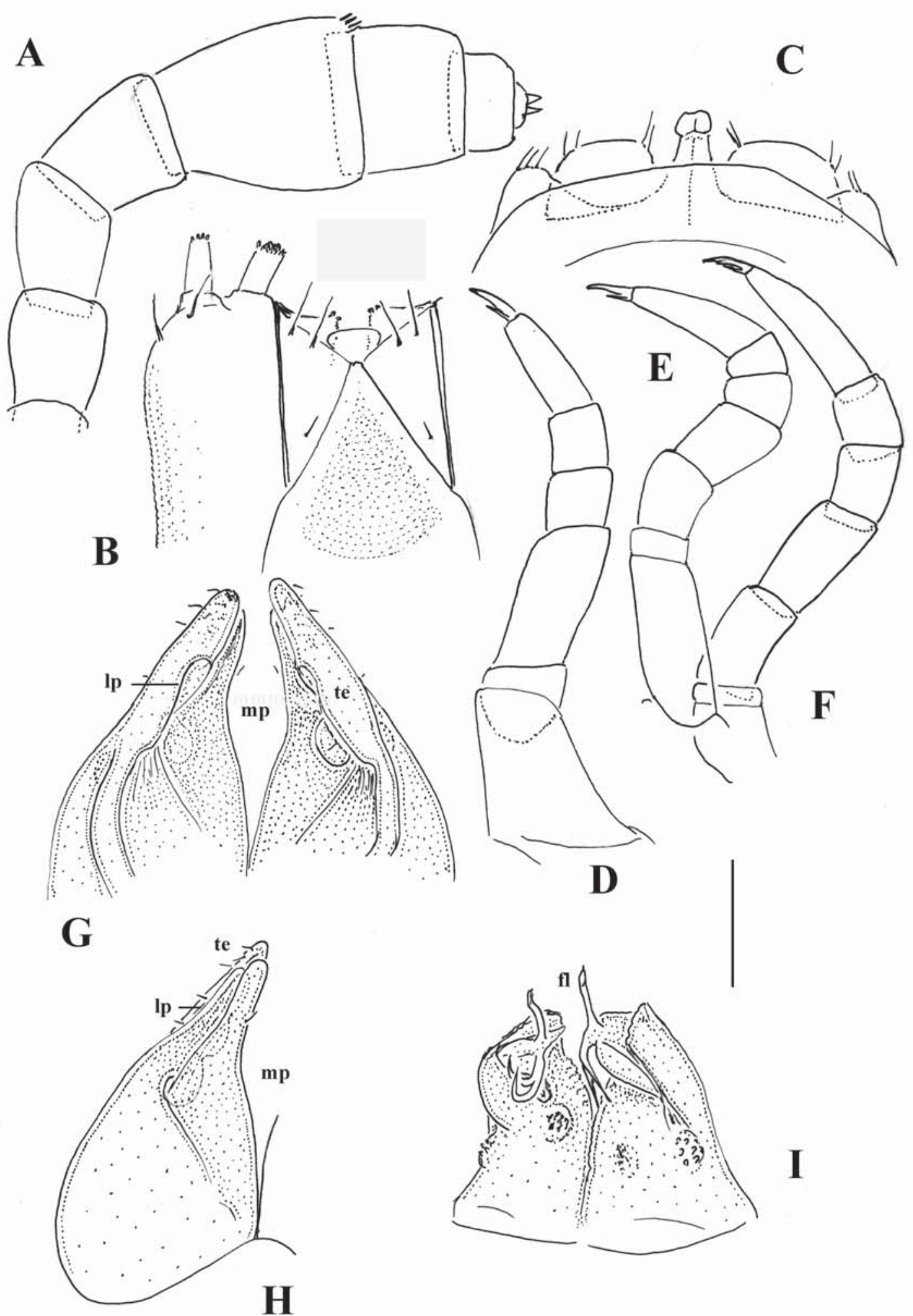

Fig. 7. Trachyjulus tjampeanus (Attems, 1903), $\mathrm{O}^{\top}$ from Cave Goa Jeruk (No. 1322). A — antenna, lateral view; B — gnathochilarium, ventral view; C - legs 1, front view; D - leg 2, caudal view; E - leg 3, caudal view; F - midbody leg, caudal view; G \& H - anterior gonopods, caudal and front views, respectively; I - posterior gonopods, caudal view. Scale bar: $0.1 \mathrm{~mm}$.

Рис. 7. Trachyjulus tjampeanus (Attems, 1903), О7 из пещеры Goa Jeruk (No. 1322). А - антенна, сбоку; В — гнатохилярий, снизу; С — ноги 1, спереди; D - нога 2, сзади; Е — нога 3, сзади; F — среднетуловищная нога, сзади; G, Н — передние гоноподы, соответственно сзади и спереди; I - задние гоноподы, сзади. Масштаб: 0,1 мм. 

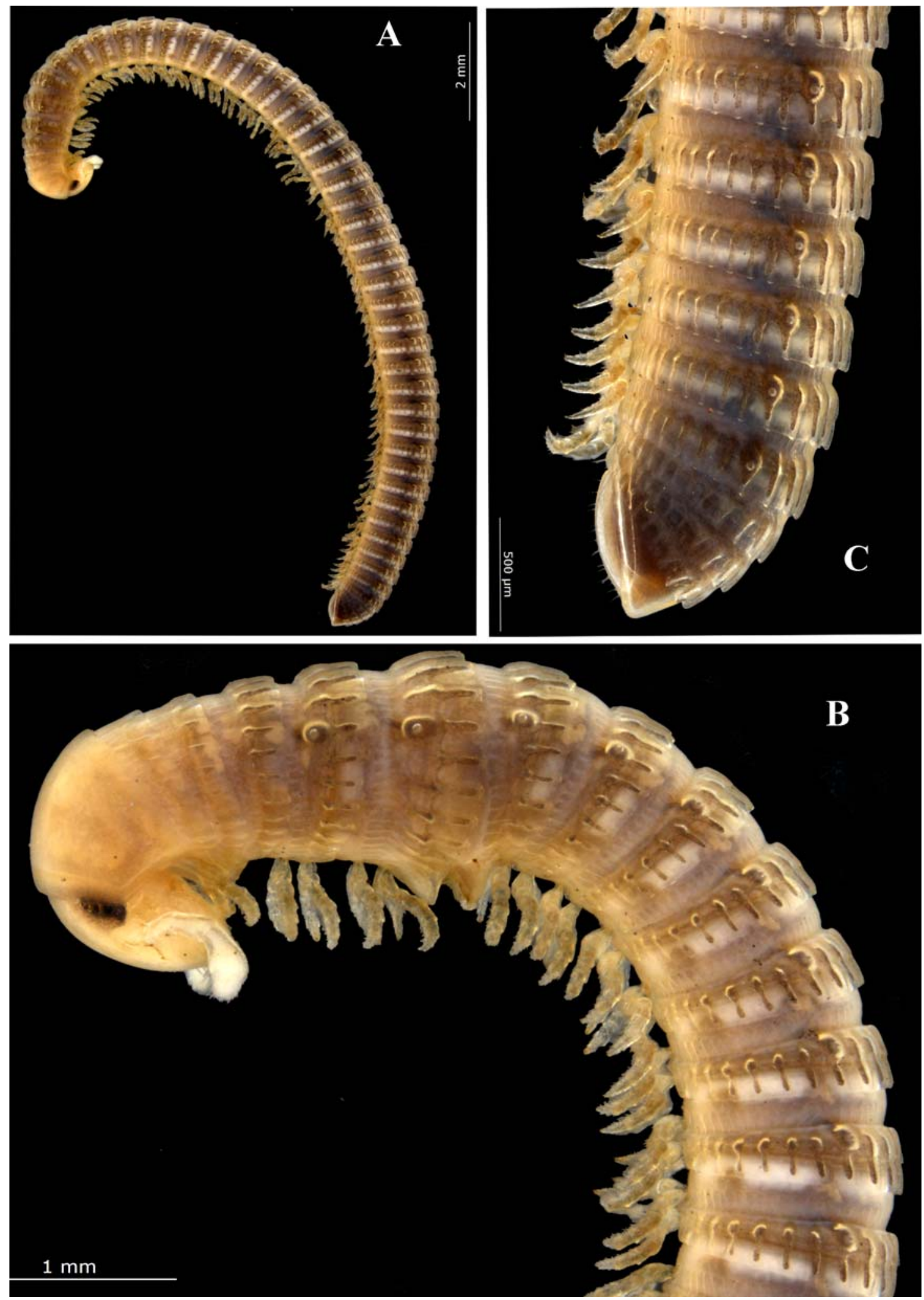

Fig. 8. Trachyjulus calvus (Pocock, 1893), O paratype of T. nordquisti ambiguus Mauriès, 1983. A - habitus, lateral view; B enlarged anterior body portion, lateral view; $\mathrm{C}$ - enlarged posterior body portion, lateral view. Photographs courtesy N. Akkari \& H. Enghoff (ZMUC).

Рис. 8. Trachyjulus calvus (Pocock, 1893), паратип О по подвида T. nordquisti ambiguus Mauriès, 1983. А — габитус, сбоку; В увеличенная передняя часть тела, сбоку; C - увеличенная задняя часть тела, сбоку. Фотографии любезно представлены N. Akkari \& H. Enghoff (ZMUC). 


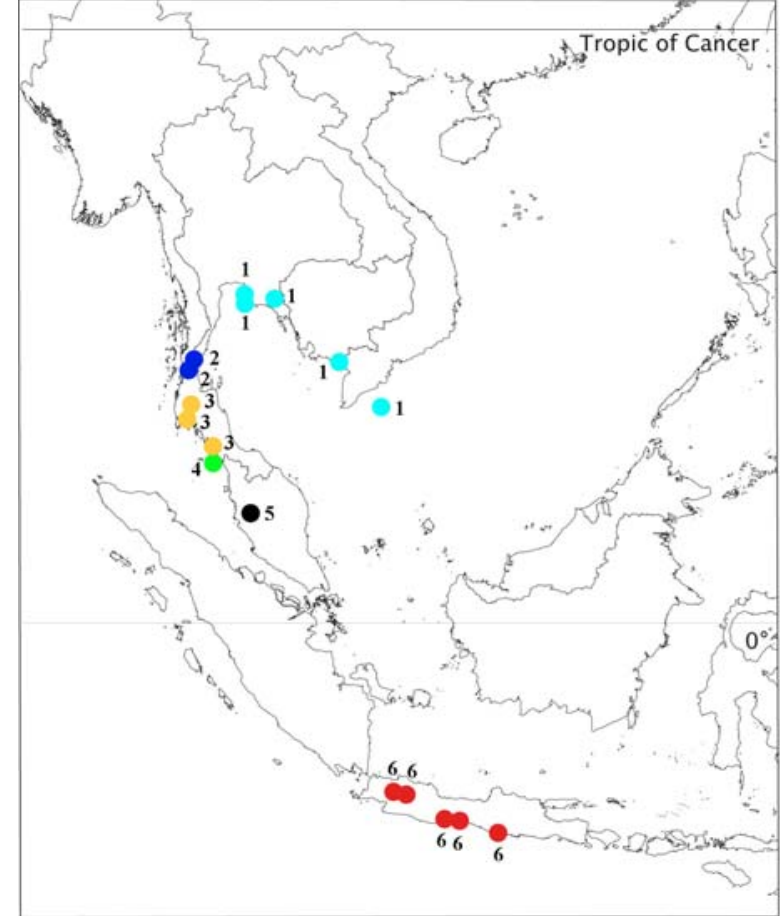

Map. Distribution of some Trachyjulus species: $1-T$. singularis; $2-T$. phylloides sp.n.; $3-T$. unciger sp.n.; $4-T$. subcalvus sp.n.; $5-$ T. beroni sp.n.; $6-$ T. tjampeanus.

Карта. Распространение некоторых видов рода Trachyjulus: 1 - T. singularis; 2 - T. phylloides sp.n.; 3 - T. unciger sp.n.; 4 - T. subcalvus sp.n.; $5-$ T. beroni sp.n.; $6-$ T. tjampeanus.

similarly short, setose, sac-shaped telopodites and then by lower, lateral, setose bulges.

Anterior gonopods (Figs $7 \mathrm{G} \& \mathrm{H}$ ) peculiar in lateral coxal process (lp) being considerably shorter than subequal both telopodite (te) and medial coxal process ( $\mathbf{m p})$, these latter from slender to stouter and finger-shaped [cf. Attems, 1903]. Posterior gonopods (Fig. 7I ) short, stout, distally microserrate/papillate, ending in a lateral lobe and a much higher tripartite flagellum (fl).

REMARKS. Attems [1903] described this species from Cave Tjompea near Bogor, Java, later mentioning a large + topotype of $2 \mathrm{~mm}$ in diameter [Attems, 1907]. Eventually, T. tjampeanus is a common and widespread species, the only congener found to occur in caves in Java (Map). Our samples also show a fair degree of variability in body size, in the number of body segments and metatergal crests, in coloration, and even in the thickness of some of the anterior gonopod elements.

\section{Trachyjulus calvus (Pocock, 1893)}

Figs $8 \& 9$

Cambala calva Pocock, 1893: 391 (D)

Cambala calva - Pocock, 1894: $377(\mathrm{~N})$; Silvestri, 1895: 749 (R); Attems, 1914: 295 (R); Attems, 1936: 252 (R)

Cambalopsis calvus — Pocock, 1895: $363(\mathrm{~N})$; Mauriès, 1970: $514(\mathrm{~N})$

Trachyjulus calvus - Jeekel, 1963: 152 (N); Mauriès, 1983: $263(\mathrm{~N})$; Jeekel, 2004: $46(\mathrm{R})$

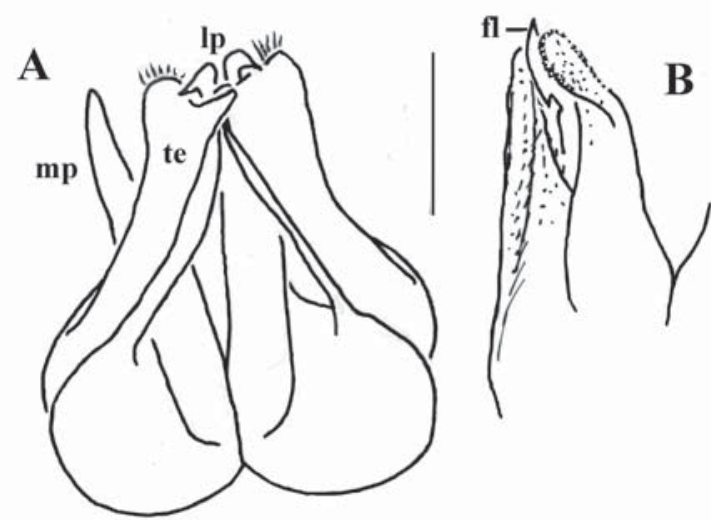

Fig. 9. Trachyjulus calvus (Pocock, 1893), O' from Ankhu Khola Valley (No. 215). A - anterior gonopods, caudal view; B right posterior gonopod, front view. Scale bar: $0.2 \mathrm{~mm}$.

Рис. 9. Trachyjulus calvus (Pocock, 1893), о из долины Ankhu Khola (No. 215). A - передние гоноподы, сзади; В правый задний гонопод, спереди. Масштаб: 0,2 мм.

Cambala calva (sic!) - Wang \& Tang, 1965: 439 (R)

Cambalopsis Nordquisti Attems, 1909: 71 (D), syn.n.

Cambalopsis nordquisti - Carl, 1912: 158 (N); Attems, 1927: 62 (R); Schubart, 1946: 396 (N)

Cambalopsis Nordquisti - Attems, 1914: 295 (R)

Trachyjulus nordquisti - Jeekel, 1963: 152, 155, $156(\mathrm{~N})$; Jeekel, 2004: 48 (R)

Trachyiulus (sic!) nordquisti ambiguus Mauriès, 1983: 262

(D), syn.n.

Trachyiulus (sic!) nordquisti - Enghoff, 1993: 214 (R)

MATERIAL. Paralectotype ${ }^{\circ}$ of Cambala calva Pocock, 1893 , following lectotype designation by Mauriès [1970] (MNHN GA 007), Burma, Palon, 1885-1889, leg. L. Fea (Coll. Brölemann, 1902, ex. typ.).

OTHER MATERIAL. 1 ○', 2 o (MNHN GA 020), Nepal, Dhading Distr., Ankhu Khola Valley, Ankhu Sangu. $650 \mathrm{~m}$, cultivated land with forest remains, 24-25.07.1983, leg. J. Martens \& W. Schawaller (No. 215); 1 o (MNHN. GA 020), Dhading Distr., descent from Kordunje into Ankhu Khola Valley, 900-650 m, 24.07.1983, leg. J. Martens \& W. Schawaller (No. 214) (both samples from Nepal identified by J.-P. Mauriès, never published earlier)

DIAGNOSIS. Differs clearly from congeners by the lateral coxal process (lp) of the anterior gonopod (Fig. 9A) being very slender and strongly detatched from a slender, conical, medial coxal process (mp), but very closely attached all along to a similarly long, but thick telopodite (te), the latter crowned with a birdhead-shaped structure, coupled with $\sigma^{7}$ legs 1 typical of Trachyjulus or the javanicus-group of Glyphiulus and the posterior gonopods (Fig. 9B) showing a rather short, axe-shaped flagellum (fl) extended only slightly above two flanking, membranous, microganulate/spiculate lobules.

REMARKS. Detailed descriptions of this species are available in Attems [1909], Carl [1912] and, especially, Schubart [1946].

Already Hoffman [1977: 713] noted, "In particular the nominal species calvus and nordquisti appear to be very close if not synonymous; the former remains known only from the type locality (Reef Island near Tovoy, lower Burma) whilst the latter has been found at Singapore, on the islands of Sulawesi, Ambon, and 

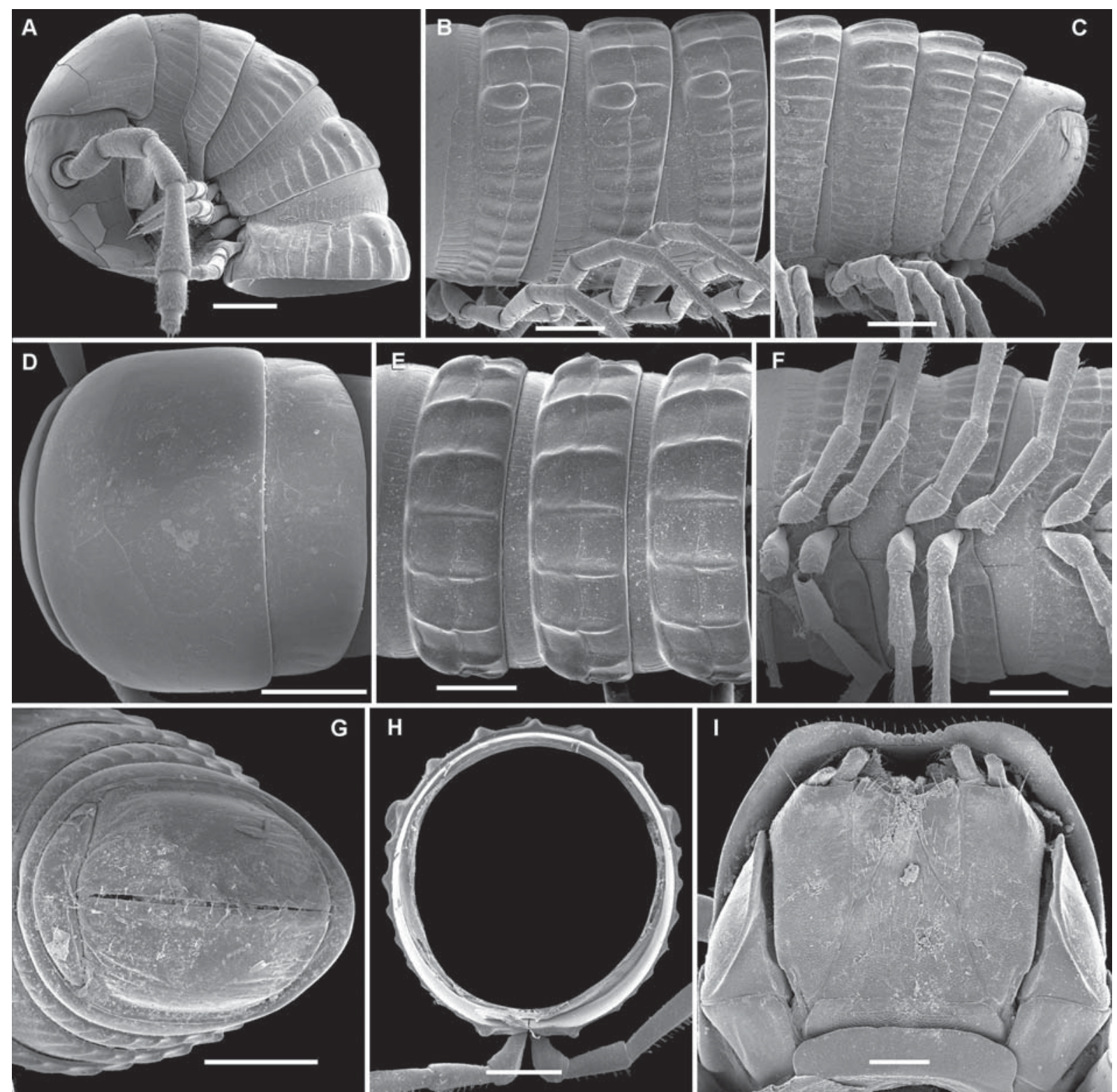

Fig. 10. Trachyjulus beroni sp.n., O7 paratype. A \& D - anterior body portion, lateral and dorsal views, respectively; B, E \& F midbody segments, lateral, dorsal and ventral views, respectively; C \& G - posterior body portion, lateral and ventral views, respectively; $\mathrm{H}$ - cross-section of a midbody segment, caudal view; G - head, ventral view. Scale bars: 0.5 (A-H ) \& $0.2 \mathrm{~mm}$ (I).

Рис. 10. Trachyjulus beroni sp.n., паратип О’. А, D - передняя часть тела, соответственно сбоку и сверху; В, Е, F среднетуловищные сегменты, соответственно сбоку, сверху и снизу; $\mathrm{C}, \mathrm{G}$ - задняя часть тела, соответственно сбоку и снизу; $\mathrm{H}-$ поперечный разрез через среднетуловищный сегмент, сзади; $\mathrm{G}$ - голова, снизу. Масштаб: 0,5 (A-H ), 0,2 мм (I).

New Britain, and in Brasil and Venezuela, clearly a successful synanthropic species". Similarly, Mauriès [1983] found only very small differences in gonopod structure of his ambiguus from the nominate form, well admitting their possible synonymy. Having carefully compared the available material, descriptions and illustrations (see also Fig. 8), as well as considering the usually broad variation range shown by several Trachyjulus species, we do not hesitate to formally synonymize $T$. nordquisti and $T$. nordquisti ambiguus with T. calvus, a medium-sized (10-25 mm long) nearly pantropical species with 4-5+4-5 blackish ocelli.

\section{Trachyjulus beroni sp.n.}

Figs 10-12.

MATERIAL. Holotype $\sigma^{7}$ (NMNHS), Malaysia, Perak State, Ipoh, Cave Gua Tempurung, 16.11.2009, leg. P. Beron \& A. Popov. Paratypes: $9 \sigma^{7} \sigma^{7}, 5$ 우, 3 juv. (NMNHS), $2 \sigma^{7} \sigma^{7}, 2$ 우 (MNHN

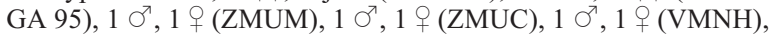
same locality, together with holotype.

NAME. Honours Petar Beron, one of the collectors.

DIAGNOSIS. Differs from congeners by the much larger size, a few troglomorphic characters like longer antennae and legs, an unpigmented or only poorly pigmented body (like in $T$. unciger sp.n.), combined 

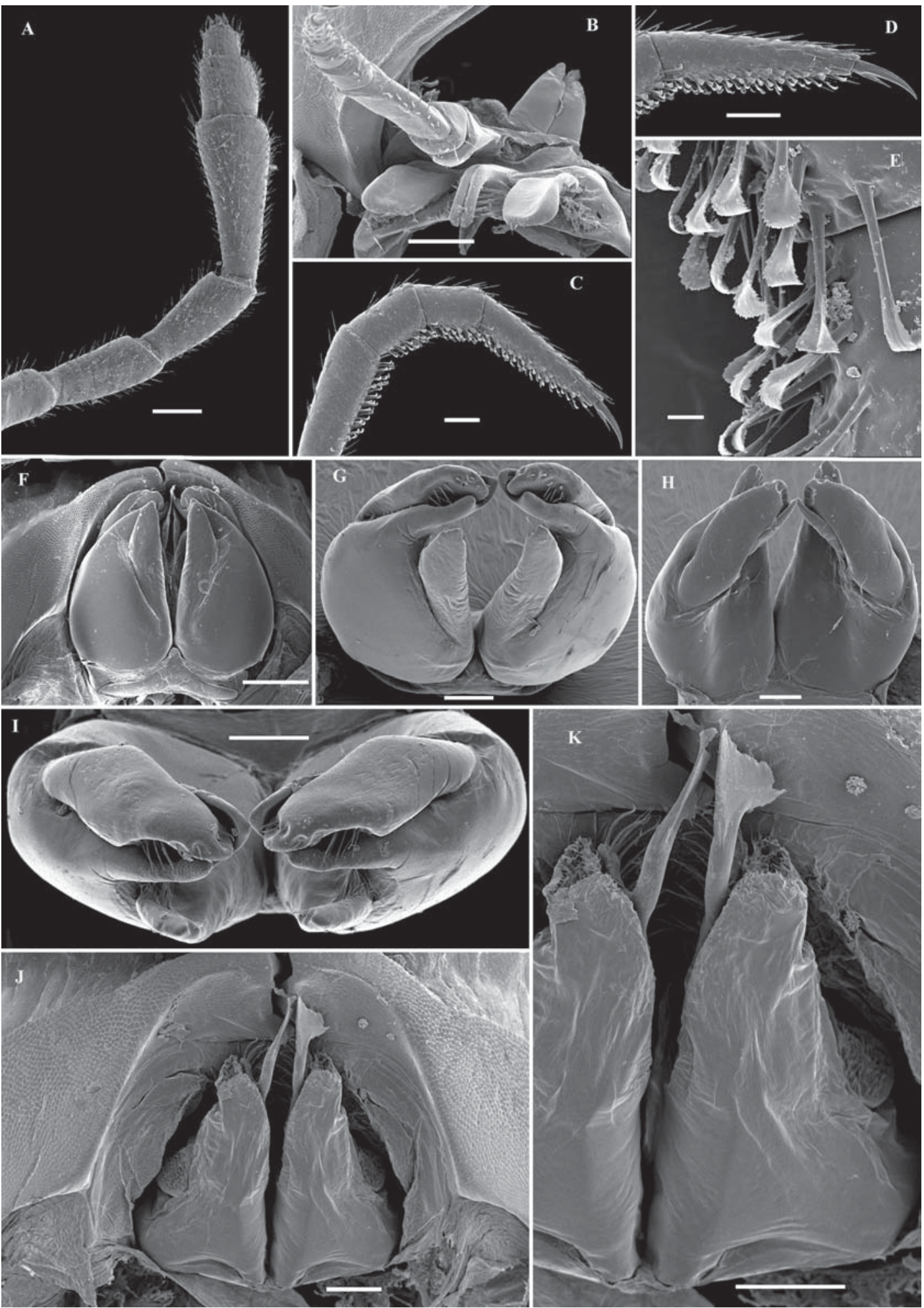

Fig. 11. Trachyjulus beroni sp.n., O' paratype. A — antenna, lateral view; B - legs $1 \& 2$, ventral view; C - most of a midbody telopodite, caudal view; D — tarsus and claw of a midbody leg, lateral view; E — phylloid setae on tarsus; F — gonopod block, front view; G, H \& I - anterior gonopods, frontoventral, caudal and ventral views, respectively; J \& K - posterior gonopods, front view. Scale bars: 0.2 (A \& B), $0.1(\mathrm{C}, \mathrm{D}, \mathrm{F}-\mathrm{K}) \& 0.01 \mathrm{~mm}(\mathrm{E})$.

Рис. 11. Trachyjulus beroni sp.n., паратип О7. А - антенна, сбоку; В - ноги 1 и 2, снизу; С - большая часть телоподита среднетуловищной ноги, сзади; D - лапка и коготок среднетуловищной ноги, сбоку; Е — листовидные щетинки, спереди; $\mathrm{F}$ гоноподный блок, спереди; G, H, I - передние гоноподы, соответственно одновременно спереди и снизу, сзади и снизу; J, K задние гоноподы, спереди. Масштаб: 0,2 (A, B), 0,1 (C, D, F-K), 0,01 мм (Е). 


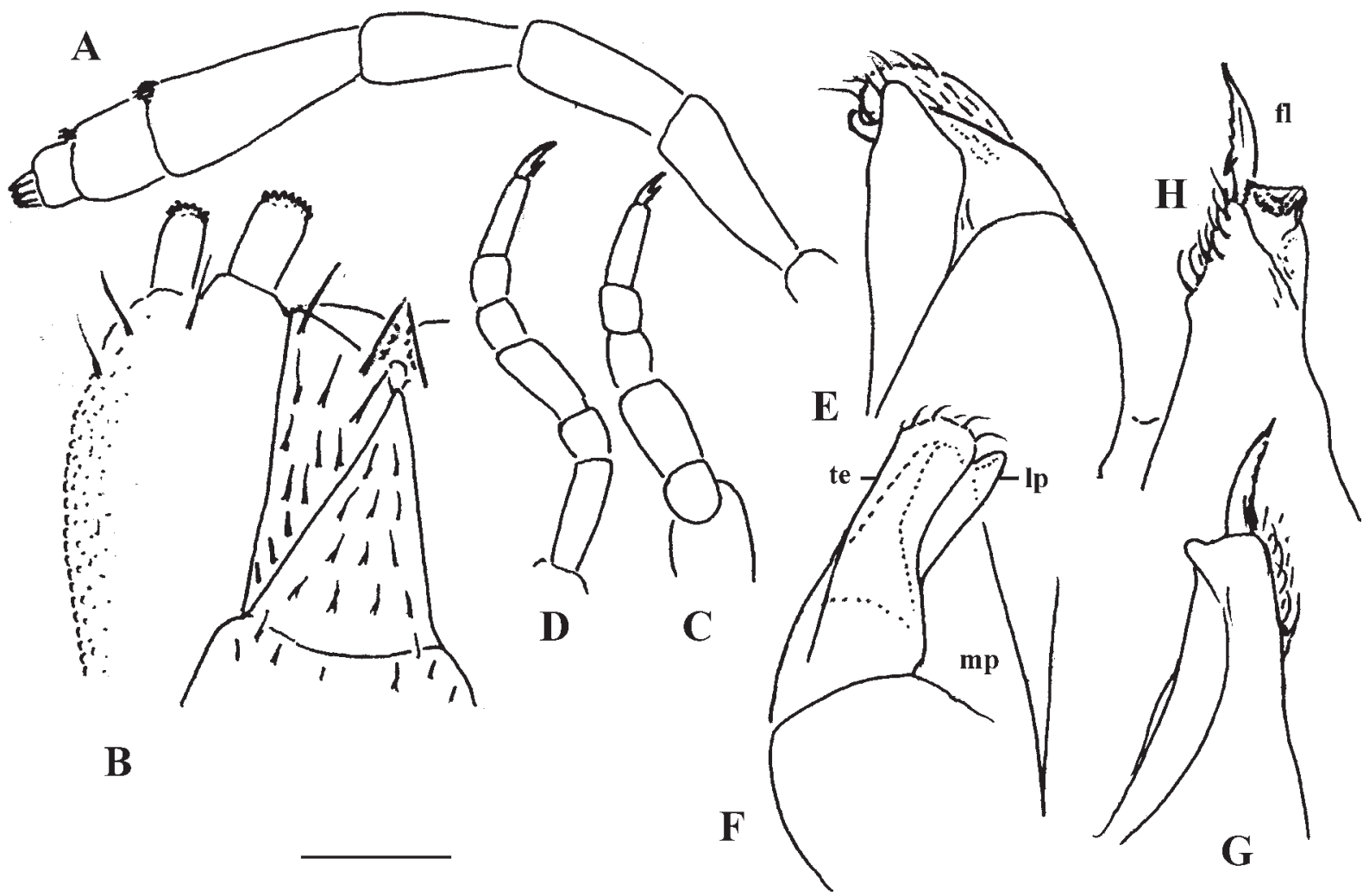

Fig. 12. Trachyjulus beroni sp.n., o paratype. A — antenna, lateral view; B - gnathochilarium, ventral view; C - leg 2, caudal view; D - leg 3, caudal view; E \& F - anterior gonopod, front and caudal views, respectively; G \& H - posterior gonopod, caudal and front views, respectively. Scale bar: $0.5(\mathrm{C}-\mathrm{H}) \& 0.2 \mathrm{~mm}(\mathrm{~A} \& \mathrm{~B})$.

Рис. 12. Trachyjulus beroni sp.n., паратип О7. А - антенна, сбоку; В - гнатохилярий, снизу; С - нога 2, сзади; D - нога 3, сзади; E, F - передний гонопод, соответственно спереди и сзади; G, H - задний гонопод, соответственно сзади и спереди. Масштаб: 0,5 (C-H), 0,2 мм (A, B).

with a strongly polytrichous gnathochilarium; the lateral coxal process of the anterior gonopods is rather strong, stout, subequal in length and width to both telopodite and medial coxal process, and evidently subtending the telopodite apex.

DESCRIPTION. Length of adults ca $20-46 \mathrm{~mm}$ $\left(\sigma^{7},+\right)$; width of midbody segments $1.3-2.0\left(\sigma^{7}\right)$ or 1.6-2.4 mm (+). Adult body with 41-73p+4-2a+T $\left(\mathrm{O}^{7}\right)$ or $44-64 \mathrm{p}+5-2 \mathrm{a}+\mathrm{T}(+)$ segments. Holotype ca $36 \mathrm{~mm}$ long and $2.0 \mathrm{~mm}$ wide, with $52 \mathrm{p}+2 \mathrm{a}+\mathrm{T}$ segments. Coloration in adults rather uniformly light grey-brown, rarely nearly pallid (smaller specimens) to castaneous brown, without a clear-cut pattern. Ocelli brown to blackish.

Usually $5+5$ ocelli in a single vertical row, variation being $4-6+4-6$, obviously regardless of sex, with $6+6$ being observed only in largest specimens. Labrum with seven small teeth medially (Fig. 10E). Antennae rather long and slender (Figs 10A, 11A, 12A). Gnathochilarium (Figs 10I \& 12B) polytrichous, mentum divided or single. Collum (Figs 10A \& D) smooth, only near lateral edge with a few light, short, superficial striae. Segments 2 and 3 also striolate to striate, following metaterga cristate, carinotaxy pattern of mid- body segments typically $10-9 / 10-9+\mathrm{I} / \mathrm{i}+2 / 2+\mathrm{m} / \mathrm{m}$ (Fig. $10 \mathrm{~A}-\mathrm{C}, \mathrm{E} \& \mathrm{H})$. Postcollum constriction evident, but not particularly strong (Fig. 10A). Midbody segments slightly compressed laterally, faintly ovoid in crosssection (Fig. 10H). Telson bare and smooth, paraprocts densely setose (Fig. 10C \& G).

Legs rather long and slender, a little longer than midbody height (Fig. 10B, C, F \& H). Ventral sides of $\sigma^{7}$ telopoditomeres beset with peculiar phylloid to plumose setae (Figs 11C-E). A short filament/spine at base of claw up to half the length of claw itself (Fig. 11D).

$0^{7}$ legs $1-3$ (Figs 11B, 12C \& D) same as in numerous species of the Glyphiulus javanicus-group [Golovatch et al., 2007b, 2011c]; legs 1 with an unciform, rather short, doubled, central process flanked by similarly short, setose, sac-shaped, flattened telopodites and then by lower, lateral, setose bulges.

Anterior gonopods (Figs 11F-I, 12E \& F) peculiar in lateral coxal process (lp) being strong, subequal in length and width to telopodite (te) and medial coxal process ( $\mathbf{m p}$ ), Ip clearly subtending te apex. Posterior gonopods (Figs 11J, K, 12G, H) shorter, distally microserrate/papillate, ending in a lateral lobe and a 

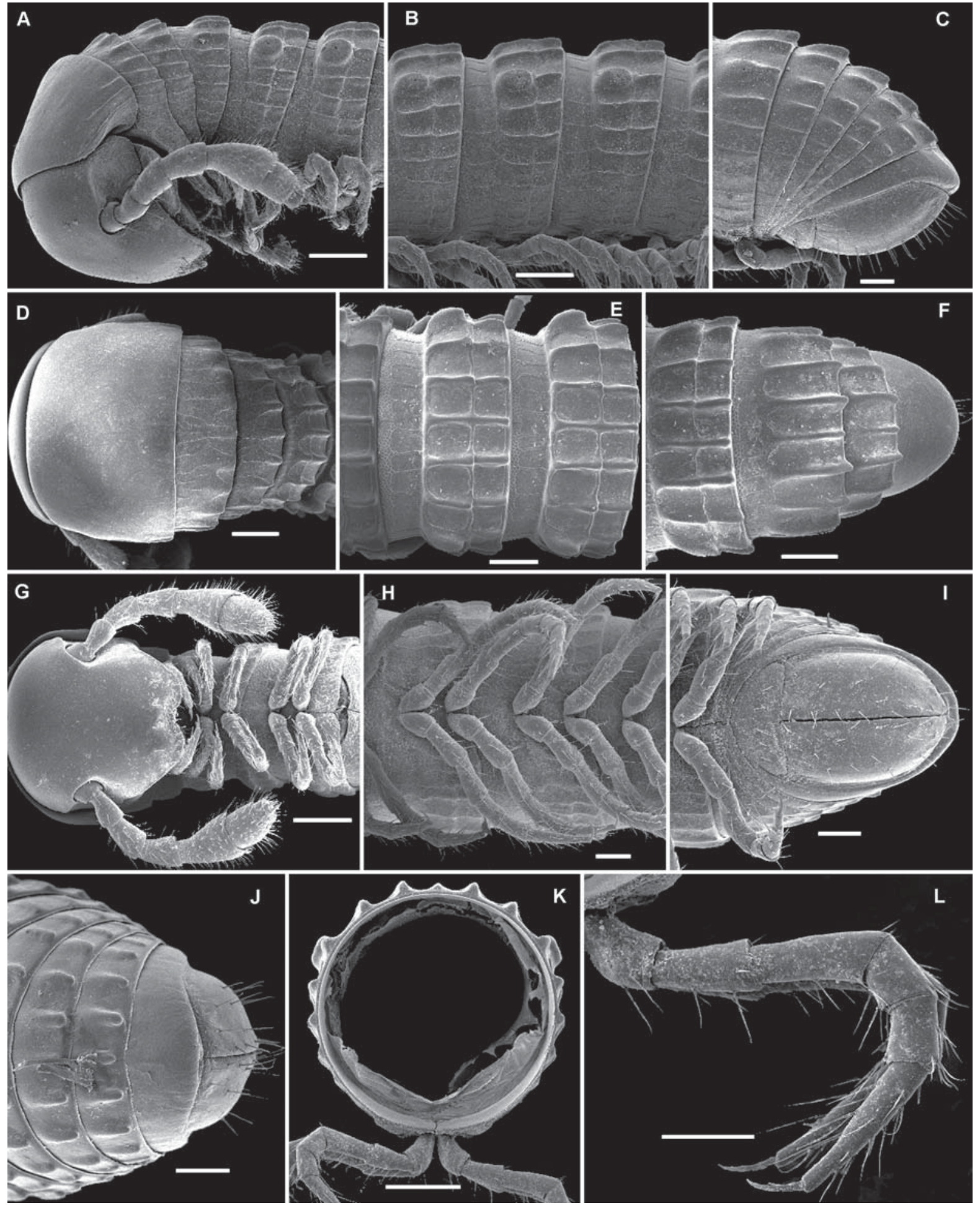

Fig. 13. Trachyjulus unciger sp.n., 9 (A-C, G-L) \& $O^{7}$ (D-F) paratypes from Cave Tham Nam (THA-PAG-036) and Cave Tham Phet (THA-PAG-032), respectively. A, D \& G - anterior body portion, lateral, dorsal and ventral views, respectively; B, E \& H - midbody segments, lateral, dorsal and ventral views, respectively; C, F, I \& J - posterior body portion, lateral, dorsal, ventral and dorsocaudal views, respectively; $\mathrm{K}$ - cross-section of a midbody segment, caudal view; L — midbody legs, caudal view. Scale bars: 0.2 (A, B, D, E, G \& K) \& $0.1 \mathrm{~mm}(\mathrm{C}, \mathrm{F}-\mathrm{I} \& \mathrm{~L})$.

Рис. 13. Trachyjulus unciger sp.n., паратипы 9 (A-C, G-L) и $0^{\top}$ (D-F) соответственно из пещеры Tham Nam (THA-PAG-036) и пещеры Tham Phet (THA-PAG-032). A, D, G - передняя часть тела, соответственно сбоку, сверху и снизу; B, E, Н - среднетуловищные сегменты, соответственно сбоку, сверху и снизу; С, F, I, J - задняя часть тела, соответственно сбоку, сверху, снизу и одновременно сверху и сзади; $\mathrm{K}$ - поперечный разрез через среднетуловищный сегмент, сзади; $\mathrm{L}$ - среднетуловищной ноги, сзади. Масштаб: 0,2 (A, B, D, E, G, K), 0,1 мм (C, F-I, L). 

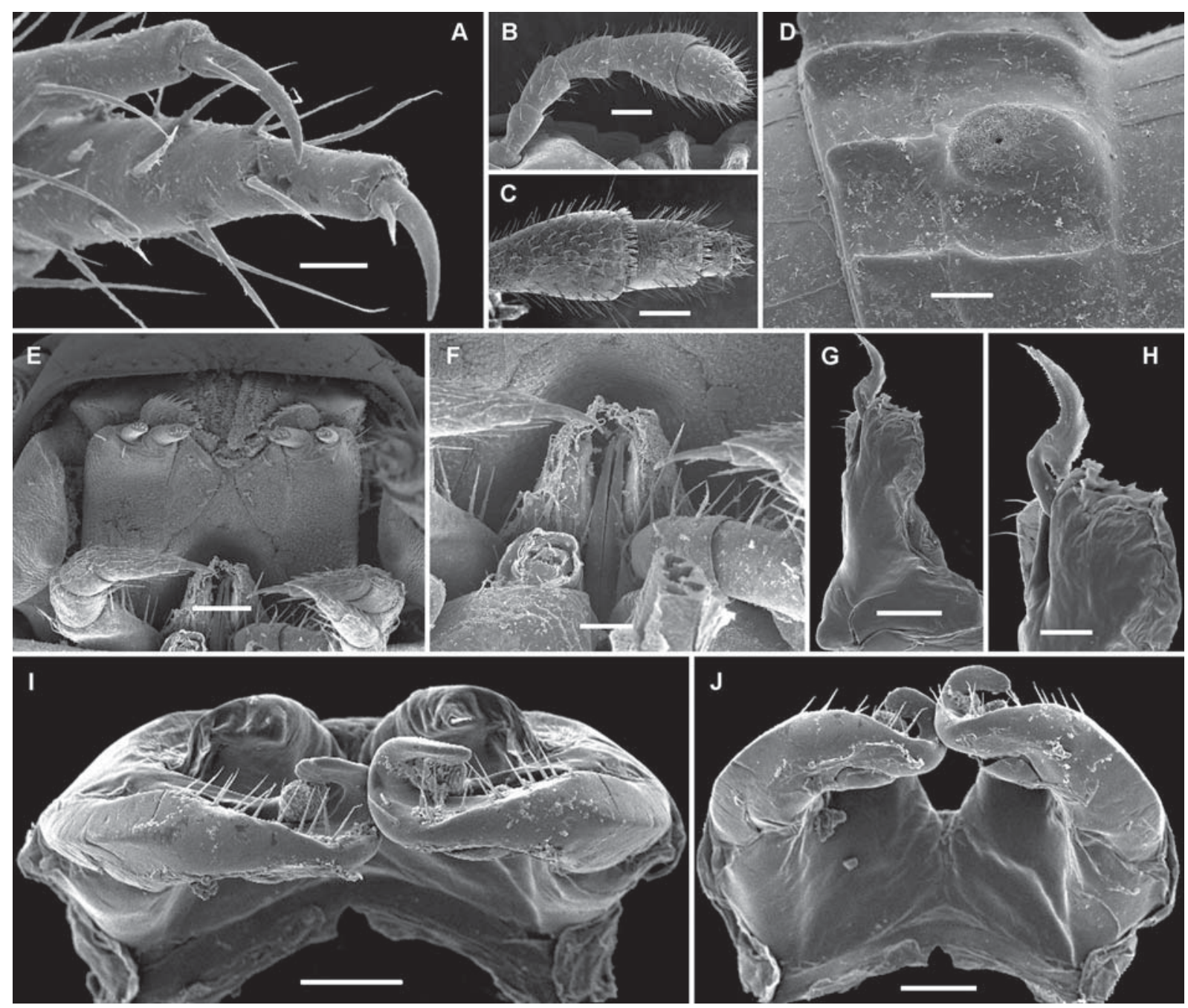

Fig. 14. Trachyjulus unciger sp.n., O (A, B, D, G-J) \& $q$ (C, E, F) paratypes from Cave Tham Nam (THA-PAG-036) and Cave Tham Phet (THA-PAG-032), respectively. A - claws, subcaudal view; B \& C - antenna and its distal part, lateral and dorsal views, respectively; D -fine texture of a midbody segment, dorsal view; E - gnathochilarium with legs 1 and 2, caudal view; F - legs 1, caudal view; G \& H - right posterior gonopod and its tip, caudal view; I \& J - anterior gonopods, ventral and caudal views, respectively. Scale bars: 0.1 (B, C \& E), 0.05 (D, F, G, I \& J) \& $0.02 \mathrm{~mm}(\mathrm{~A} \& \mathrm{H})$.

Рис. 14. Trachyjulus unciger sp.n., паратипы О (A, B, D, G-J) и + (C, E, F) соответственно из пещеры Tham Nam (THA-PAG036) и пещеры Tham Phet (THA-PAG-032). А — коготки, почти сзади; В, С - антенна и ее дистальная часть, соответственно сбоку и сверху; D - тонкая текстура покровов среднетуловищного сегмента, сверху; Е — гнатохилярий с ногами 1 и 2, сзади; F — ноги 1, сзади; G, H - правый задний гонопод и его вершина, сзади; I, J — передние гоноподы, соответственно снизу и сзади. Масштаб: 0,1 (B, C, E), 0,05 (D, F, G, I, J), 0,02 мм (A, H).

considerably higher, axe-shaped to phylloid flagellum (fl).

REMARKS. Because this new species shows a few troglomorphic traits, it might well be a troglobite. So far as known, it occurs in a single cave (Map).

\section{Trachyjulus unciger sp.n.}

Figs 13-18.

MATERIAL. Holotype $\sigma^{7}$ (MNHN GA 96), Thailand, Phangnga Prov., Phangnga, Thap Put, Cave Tham Nam, 20.07.1987, leg. Ph. Leclerc (THA-PAG-036). Paratypes: $3 O^{7} O^{\top}, 11$ ㅇ (MNHN GA 96), $10^{7}, 1$ (ZMUM), 2 우 (SEM), same locality, together with holotype; $2 O^{7} \sigma^{7}, 2$ 우, 2 juv. (MNHN GA 96), $1 \sigma^{7}$ (SEM), Thailand, Phangnga Prov., Phangnga, Thap Put, Cave Tham Phet,
18.07.1987, leg. Ph. Leclerc (THA-PAG-032); $1 \sigma^{\top 7}$ (MNHN GA 96), $10^{7}$ (MZCU), 1 \% (SEM), Phangnga Prov., Phangnga, Cave Tham Poung Chang, 27.07.1985, leg. C. Dutin (THA-KP-003); 1 $0^{7}, 1$ ㅇ, 1 fragm., 2 juv. (MNHN GA 96), $10^{7}, 1$ ㅇ (ZMUC), 1 ㅇ (SEM), Surat Thani Prov., Phanom, Cave Tham Khao Sok 1, 25.07.1987, leg. L. Deharveng (THA-SUT-018); $10^{7}, 1+$ (MNHN GA 96), 1 ( (SEM), same cave, guano, berleseate, 25.07.1987, leg. L. Deharveng (THA-SUT-021); $2 \mathrm{O}^{7} \mathrm{O}^{7}, 2$ 우 (VMNH), 1 (SEM), Satun Prov., Manang Distr., Cave Tham Phu Pha Phet, $07^{\circ} 07.683^{\prime}$, $099^{\circ} 59.717^{\prime} \mathrm{E}, 05.01 .2001$, leg. H. Steiner.

NAME. To reflect the unusual shape of $\sigma^{7}$ legs 1 showing a large central uncus flanked by large, abundantly setose, sac-shaped telopodites, just like those observed in most of the species of Plusioglyphiulus Silvestri, 1923 [Golovatch et al., 2011a]. A similar structure of $\sigma^{r}$ legs 1 among known Trachyjulus spe- 


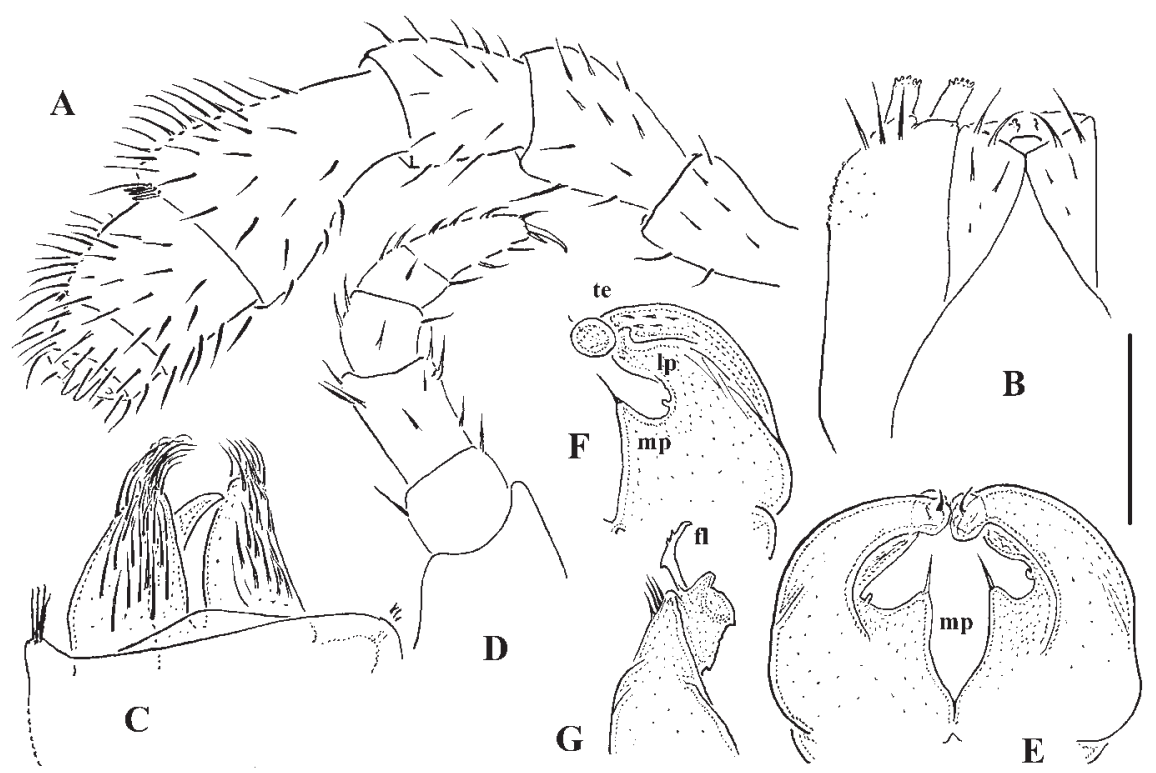

Fig. 15. Trachyjulus unciger sp.n., O' paratype from Cave Tham Nam (THA-PAG-036). A - antenna, lateral view; B - gnathochilarium, ventral view; C - legs 1, frontolateral view; D - leg 2, caudal view; E \& F - anterior gonopod, front and caudal views, respectively; $\mathrm{G}$ - left posterior gonopod, front view. Scale bar: $0.2 \mathrm{~mm}$.

Рис. 15. Trachyjulus unciger sp.n., паратип О7 из пещеры Tham Nam (THA-PAG-036). A — антенна, сбоку; В — гнатохилярий, снизу; С — ноги 1, одновременно спереди и сбоку; D — нога 2, сзади; Е и F — передний гонопод, соответственно спереди и сзади; $\mathrm{G}$ - левый задний гонопод, спереди. Масштаб: 0,2 мм.

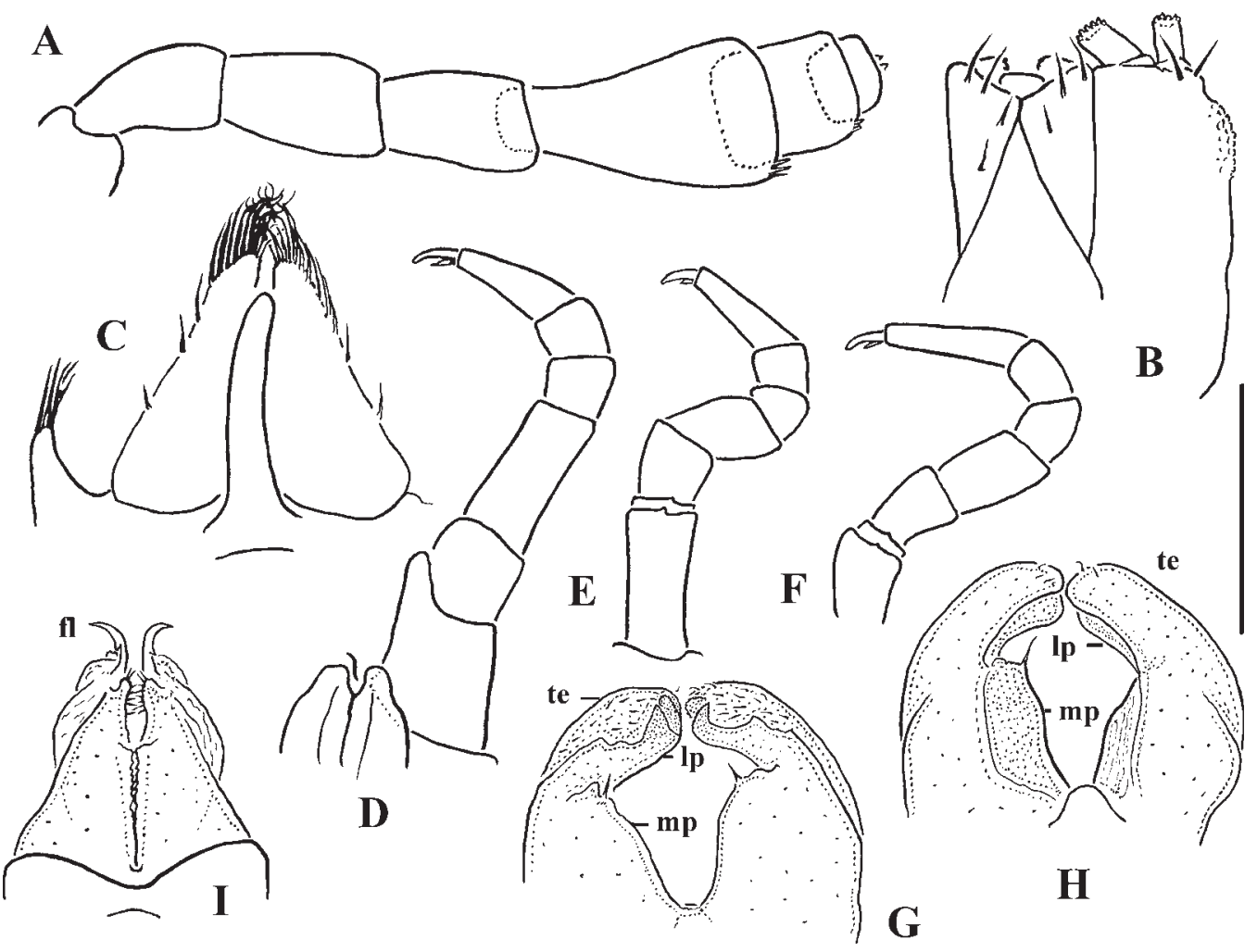

Fig. 16. Trachyjulus unciger sp.n., $\mathrm{O}^{7}$ paratype from Cave Tham Poung Chang (THA-KP-003). A - antenna, lateral view; B gnathochilarium, ventral view; C - legs 1, caudal view; D - leg 2, caudal view; E - leg 3, caudal view; F — midbody leg, caudal view; $\mathrm{G} \& \mathrm{H}$ - anterior gonopods, front and caudal views, respectively; I — posterior gonopods, caudal view. Scale bar: $0.4 \mathrm{~mm}$.

Рис. 16. Trachyjulus unciger sp.n., паратип О из пещеры Tham Poung Chang (ТНА-KP-003). А — антенна, сбоку; В гнатохилярий, снизу; С - ноги 1, сзади; D - нога 2, сзади; Е - нога 3, сзади; F - среднетуловищная нога, сзади; $\mathrm{G}, \mathrm{H}-$ передние гоноподы, соответственно спереди и сзади; I - задние гоноподы, сзади. Масштаб: 0,4 мм. 


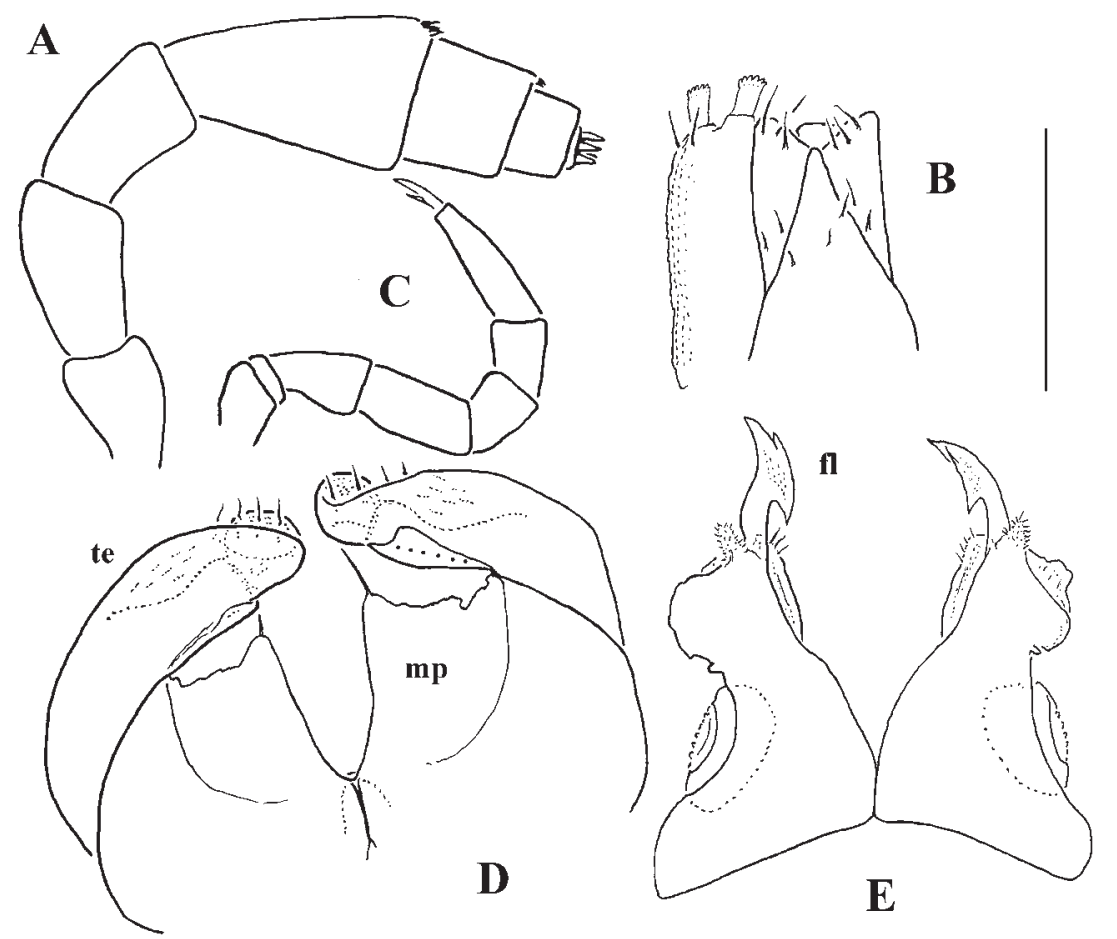

Fig. 17. Trachyjulus unciger sp.n., $O^{T}$ paratype from Cave Tham Khao Sok 1 (THA-SUT-018). A — antenna, lateral view; B gnathochilarium, ventral view; C - leg 2, caudal view; D - anterior gonopods, caudal view; E - posterior gonopod, caudal view. Scale bar: $0.4(\mathrm{~A}-\mathrm{C}) \& 0.2 \mathrm{~mm}(\mathrm{D} \& \mathrm{E})$.

Рис. 17. Trachyjulus unciger sp.n., паратип Оㄱ из пещеры Tham Khao Sok 1 (THA-SUT-018). A — антенна, сбоку; В гнатохилярий, снизу; C - ноги 1, сзади; D - передние гоноподы, сзади; Е - задний гонопод, сзади. Масштаб: 0,4 (A-C) и 0,2 мм $(\mathrm{D}, \mathrm{E})$.

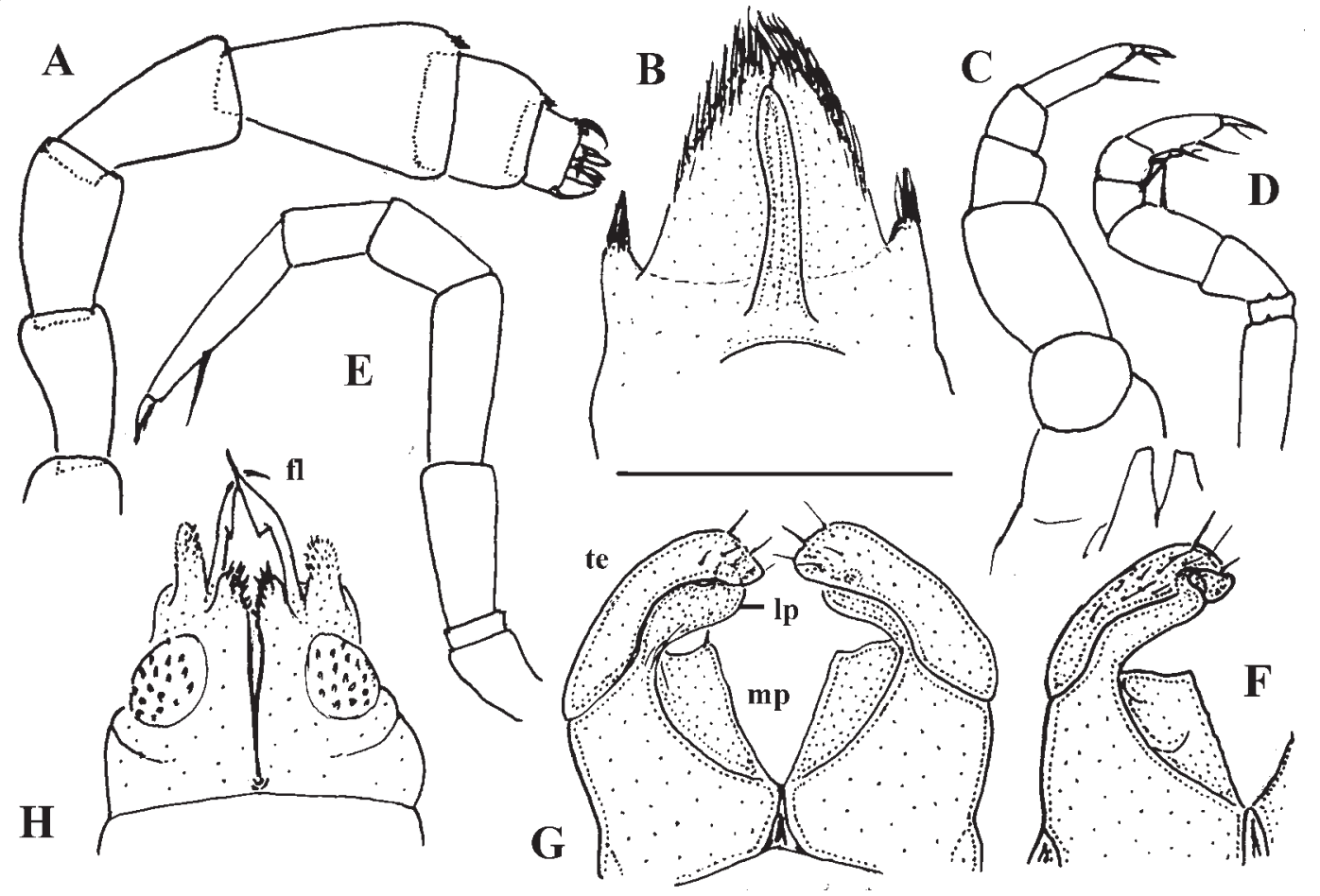

Fig. 18. Trachyjulus unciger sp.n., $0^{7}$ paratype from Cave Tham Phu Pha Phet. A — antenna, lateral view; B — legs 1, caudal view; C - leg 2, caudal view; D - leg 3, caudal view; E - midbody leg, caudal view; F \& G - anterior gonopods, front and caudal views, respectively; $\mathrm{H}$ - posterior gonopods, front view. Scale bar: $0.4 \mathrm{~mm}$.

Рис. 18. Trachyjulus unciger sp.n., паратип О из пещеры Tham Phu Pha Phet. A — антенна, сбоку; В — ноги 1, сзади; С - нога 2, сзади; D — нога 3, сзади; Е - среднетуловищная нога, сзади; F, G — передние гоноподы, соответственно спереди и сзади; Н задние гоноподы, спереди. Масштаб: 0,4 мм. 

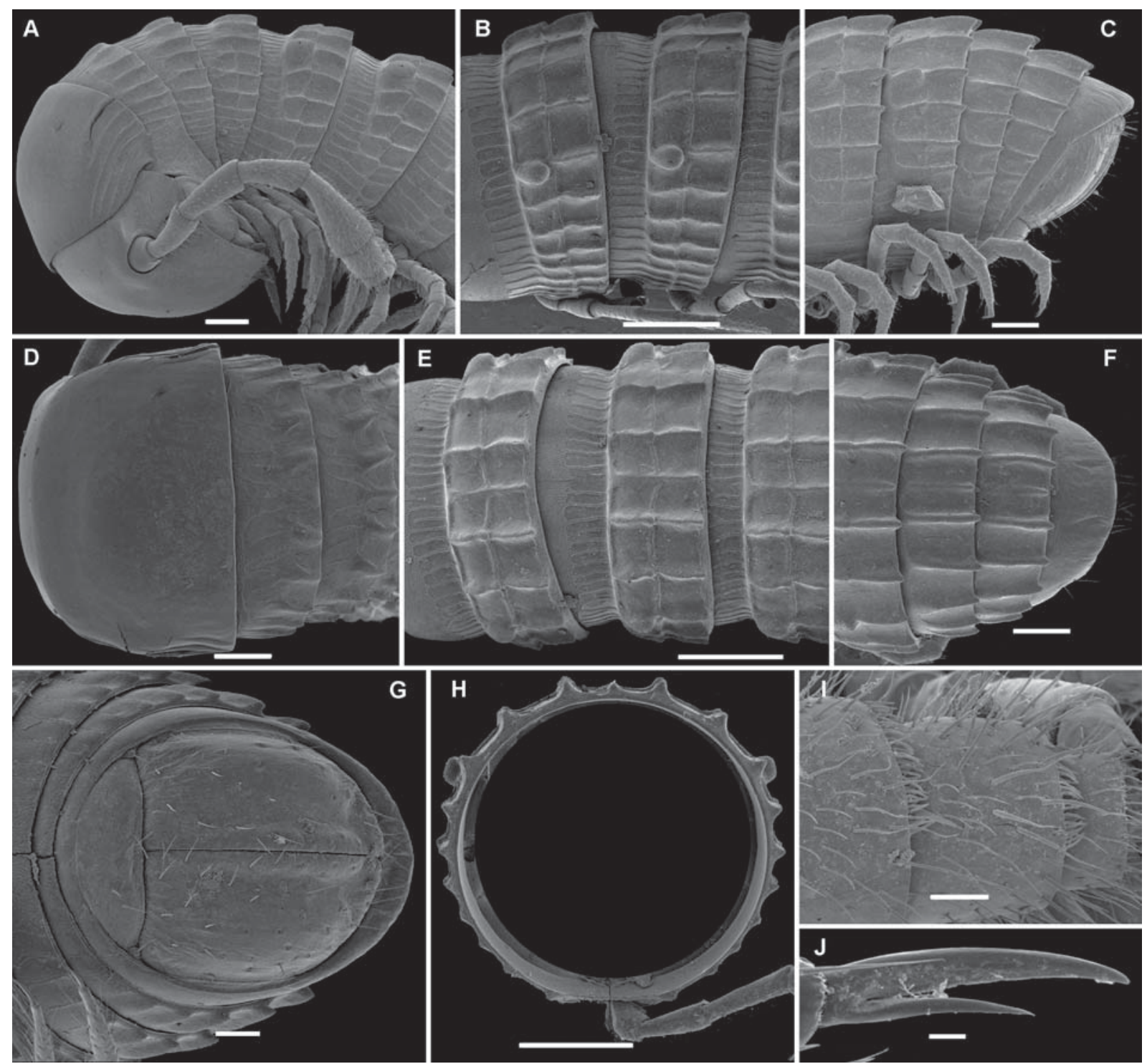

Fig. 19. Trachyjulus subcalvus sp.n., + paratype. A \& D - anterior body portion, lateral and dorsal views, respectively; B \& E midbody segments, lateral and dorsal views, respectively; C, F \& G - posterior body portion, lateral, dorsal and ventral views, respectively; $\mathrm{H}$ - cross-section of a midbody segment, caudal view; I - sensilla on antennomeres 5 and 6 , dorsal view; J - claw of a midbody leg, caudal view. Scale bars: 0.5 (B, E \& H), 0.2 (A, C, D \& F), 0.1 (G), 0.05 (I) \& $0.01 \mathrm{~mm}(\mathrm{~J})$.

Рис. 19. Trachyjulus subcalvus sp.n., + paratype. А, D - передняя часть тела, соответственно сбоку и сверху; В, Е среднетуловищные сегменты, соответственно сбоку и сверху; C, F, G - задняя часть тела, соответственно сбоку, сверху и снизу; $\mathrm{H}$ - поперечный разрез через среднетуловищный сегмент, сзади; I — сенсиллы на члениках 5 и 6 антенн, сверху; J — коготок среднетуловищной ноги, сзади. Масштаб: 0,5 (B, Е, Н), 0,2 (А, C, D, F), 0,1 (G), 0,05 (I), 0,01 мм (J).

cies seems to be observed only in T. humberti, from Sri Lanka, albeit both the central hook and lateral telopodite vestiges are somewhat shorter [Carl, 1911].

DIAGNOSIS. Differs from congeners in the peculiar, Plusioglyphiulus-like shape of $\bigcirc^{7}$ legs 1 , coupled with an oligotrichous gnathochilarium, a small, ventral, midway denticle on $\bigcirc^{7}$ genae and a particularly low, lobe-shaped, medial coxal process of the anterior gonopods, whilst the lateral coxal process is slender and evidently subtends the telopodite apex.

DESCRIPTION. Length of adults ca $23-36\left(\sigma^{7}\right)$ or 25-42 mm (+); width of midbody segments $1.2-2.0$ $\left(\sigma^{7}, 9\right)$. Adult body with $49-70 \mathrm{p}+4-2 \mathrm{a}+\mathrm{T}$ segments $\left(\mathrm{O}^{\gamma},+\right.$ ). Holotype ca $36 \mathrm{~mm}$ long and $1.8 \mathrm{~mm}$ wide, with $65 \mathrm{p}+2 \mathrm{a}+\mathrm{T}$ segments. Coloration in adults uniformly pallid to grey-brown, without a clear-cut pattern. Ocelli brown to blackish.

Invariably $4+4$ ocelli in a single vertical row. Labrum with seven small teeth medially (Fig. 14E). $\sigma^{7}$ genae with a small, low, but evident, subtriangular tooth ventrally at about midway (Fig. 14E). Antennae rather long and slender (Figs 13A, G, 14B, C, 15A, 16A, 17A, 18A). Gnathochilarium (Figs 14E, 15B, 16B, 17B) oligotrichous, mentum single. Collum (Fig. 


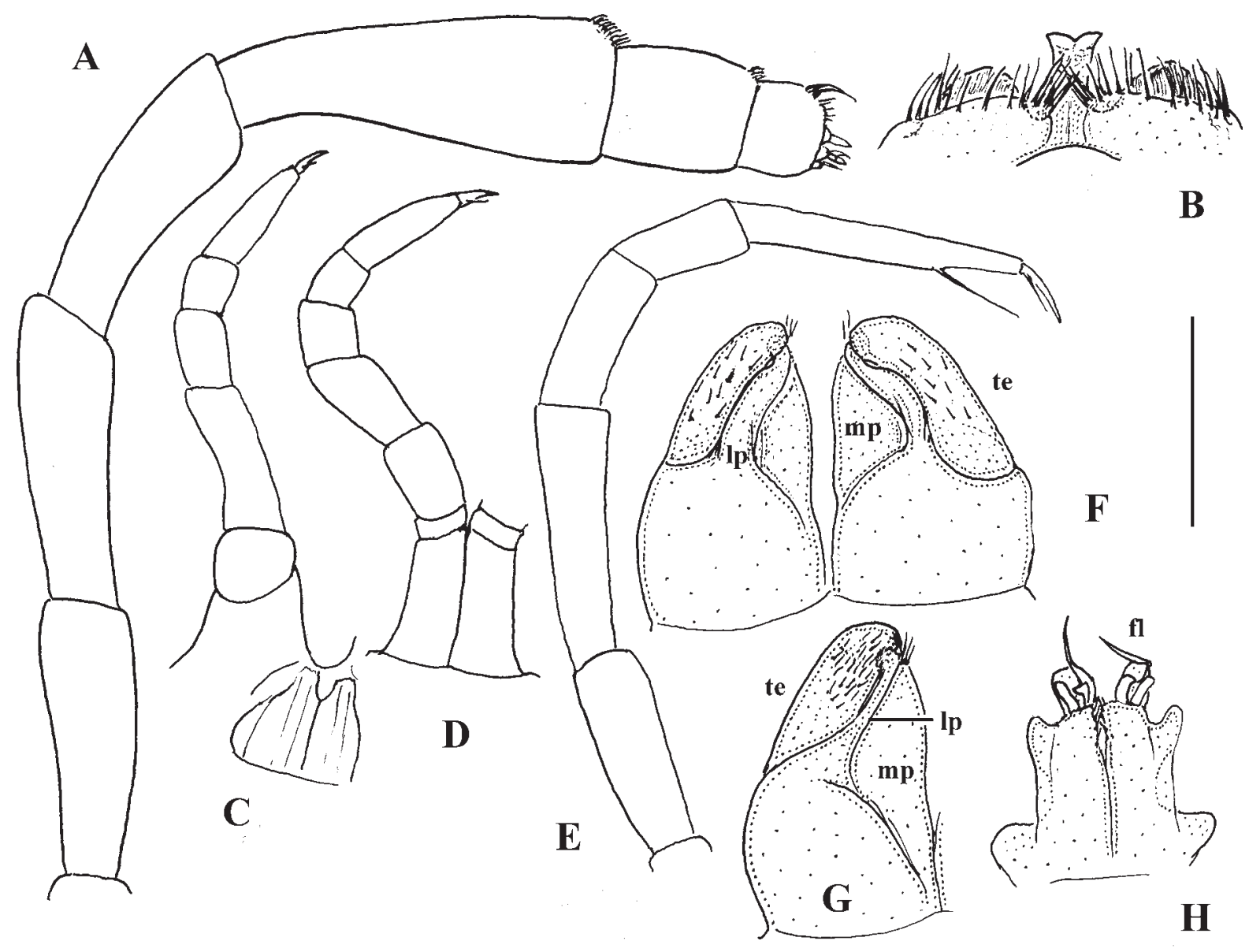

Fig. 20. Trachyjulus subcalvus sp.n., holotype. A - antenna, lateral view; B - legs 1, front view; C - leg 2, caudal view; D - leg 3, caudal view; E - midbody leg, caudal view; F \& G — anterior gonopods, caudal and front views, respectively; H — posterior gonopods, front view. Scale bar: $0.3 \mathrm{~mm}$.

Рис. 20. Trachyjulus subcalvus sp.n., голотип. А - антенна, сбоку; В — ноги 1, спереди; С — нога 2, сзади; D — нога 3, сзади; E - среднетуловищная нога, сзади; F, G - передние гоноподы, соответственно сзади и спереди; Н - задние гоноподы, спереди. Масштаб: 0,3 мм.

13A \& D) smooth, only near lateral edge with a few light, short, superficial striae. Segments 2 and 3 faintly cristate, following metaterga fully cristate, carinotaxy pattern of midbody segments typically $8-6 / 8-6+\mathrm{I} / \mathrm{i}+2 /$ $2+\mathrm{m} / \mathrm{m}$ (Figs 13A-F, J, K, 14D). Postcollum constriction evident, but not particularly strong (Fig. 13A, D \& $\mathrm{G})$. Midbody segments nearly round in cross-section (Fig. 13K). Telson bare and smooth, paraprocts densely setose (Fig. 13C, F, I \& J).

Legs rather long and slender, about as long as midbody height (Figs 13A-C, G-I, K, L, 16F, 18E). A short filament/spine at base of claw slightly exceeding half the length of claw itself (Figs 14A, 16F, 18E).

$\sigma^{7}$ legs 1 (Figs 14E, F, 15C, D, 16C-E, 17C, 18BD) same as in numerous species of Plusioglyphiulus [Golovatch et al., 2009, 2011a], i.e. with a strong central uncus placed between strong, high, densely setose, sac-shaped telopodites supplied with only very modest, densely setose, tuberculiform bulges more laterally. $\sigma^{7}$ legs 2 and 3 more like in Glyphiulus species, i.e. the former not too strongly incrassate, the latter not too strongly reduced.

Anterior gonopods (Figs 14I, J, 15E, F, 16G, H, $17 \mathrm{D}, 18 \mathrm{~F}, \mathrm{G})$ peculiar in lateral coxal process (lp) being slender and long, subequal in length to a digitiform and setose telopodite (te), clearly subtending te apex, but with a much shorter, lobe-shaped medial coxal process (mp). Posterior gonopods (Figs 14G, H, $15 \mathrm{G}, 16 \mathrm{I}, 17 \mathrm{E}, 18 \mathrm{H})$ shorter, distally microserrate/papillate, ending in a higher lateral and a shorter mesal lobe, both divided by a very high, axe-shaped flagellum.

REMARKS. This species seems to show too few troglomorphic traits (except perhaps a mostly unpigmented tegument) to be considered a candidate troglobite. Its distribution is coherent, taking up a rather small area in southern Thailand (Map).

\section{Trachyjulus subcalvus sp.n.}

Figs $19 \& 20$.

MATERIAL. Holotype $\sigma^{7}$ (VMNH), Malaysia, Kedah State, Langkawi Island, Cave Gua Tok Yed, $06^{\circ} 19.120^{\prime} \mathrm{N}, 099^{\circ} 52.812^{\prime} \mathrm{E}$, 

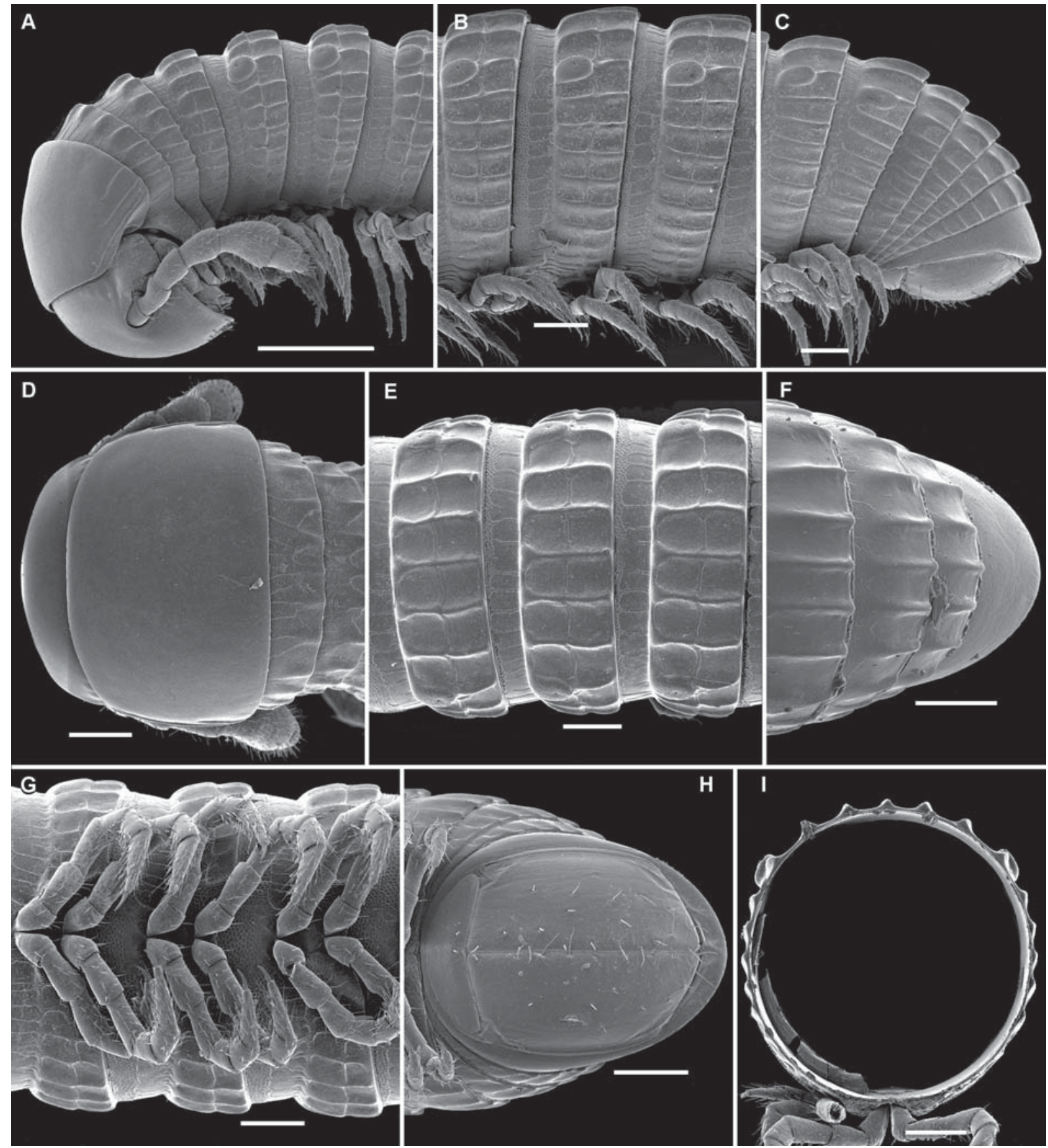

Fig. 21. Trachyjulus phylloides sp.n., O paratype from Cave Tham Phra Khayang. A \& D - anterior body portion, lateral and dorsal views, respectively; B, E \& G - midbody segments, lateral, dorsal and ventral views, respectively; C, F \& H - posterior body portion, lateral, dorsal and ventral views, respectively; I - cross-section of a midbody segment, caudal view. Scale bars: $0.2 \mathrm{~mm}$.

Рис. 21. Trachyjulus phylloides sp.n., паратип О из пещеры Tham Phra Khayang. A, D - передняя часть тела, соответственно сбоку и сверху; В, E, G - среднетуловищные сегменты, соответственно сбоку, сверху и снизу; С, F, H - задняя часть тела, соответственно сбоку, сверху и снизу; I — поперечный разрез через среднетуловищный сегмент, сзади. Масштаб: 0,2 мм.

08.01.2002, leg. H. Steiner. Paratypes: 19 (VMNS), 1 (MNHN GA 97), 1 (SEM), same locality, together with holotype.

NAME. To reflect the strong similarities in gonopod structure to T. calvus.

DIAGNOSIS. Differs from congeners by the lack of visible ocelli, coupled with a few other troglomorphic traits like longer antennae and legs, and an unpigmented body; the lateral coxal process of the anterior gonopods is slender, much like in T. calvus, subequal in length to both telopodite and medial coxal process, and evidently subtending the telopodite apex; the posterior gonopods, unlike those of T. calvus, with a longer and simpler flagellum. 

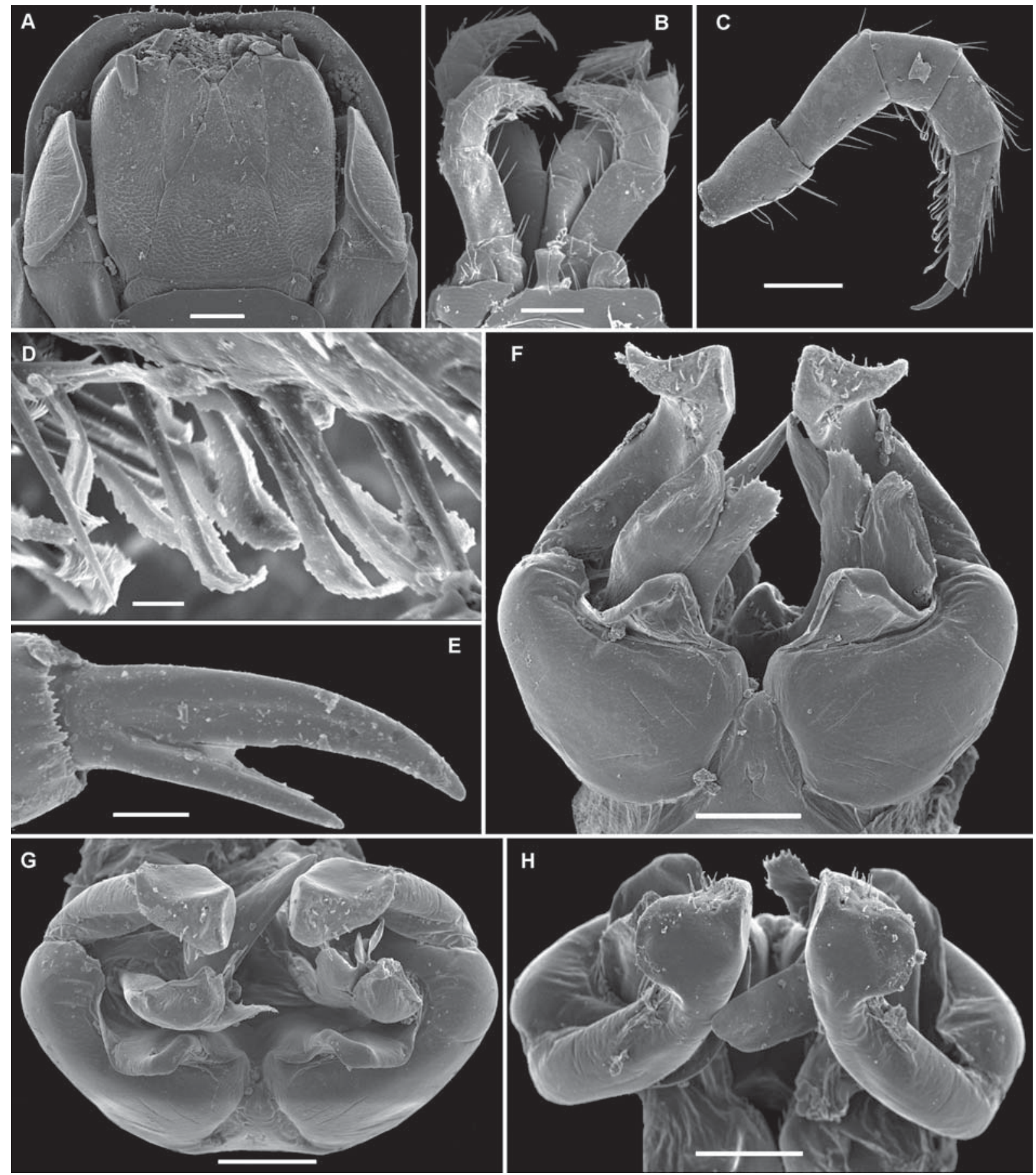

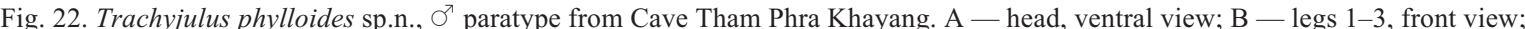
C \& D - midbody leg and enlarged phylloid setae, respectively, caudal view; E - claw of a midbody leg, caudal view; F-H - gonopod block, caudal, subventral and ventral views, respectively. Scale bars: $0.1(\mathrm{~A}-\mathrm{C}, \mathrm{F}-\mathrm{H}) \& 0.01 \mathrm{~mm}(\mathrm{D} \& \mathrm{E})$.

Рис. 22. Trachyjulus phylloides sp.n., паратип О7 из пещеры Tham Phra Khayang. A — голова, снизу; В - ноги 1-3, спереди; C, $\mathrm{D}$ - соответственно среднетуловищная нога и увеличенные листовидные щетинки, сзади; Е - коготок среднетуловищной ноги, сзади; F-H - гоноподный блок, соответственно сзади, почти снизу и снизу. Масштаб: $0,1(\mathrm{~A}-\mathrm{C}, \mathrm{F}-\mathrm{H})$ и 0,01 мм $(\mathrm{D}, \mathrm{E})$.

DESCRIPTION. Length ca 33 (holotype), 29 or 32 $\mathrm{mm}(+)$; width of midbody segments 1.6 (holotype), 1.6 or $1.7 \mathrm{~mm}(+)$. Adult body with $65 \mathrm{p}+2 \mathrm{a}+\mathrm{T}$ (holotype) or 45-48p+2a+T (+) segments. Coloration uniformly pallid, ocelli invisible.
Labrum with ca 7 small teeth medially. Antennae rather long and slender, antennomeres 5 and 6 with especially numerous sensory bacilli distodorsally (Fig. 19A, I, 20A). Gnathochilarium rather oligotrichous, mentum single. Collum (Figs 19A \& D) smooth, only laterally with 5 


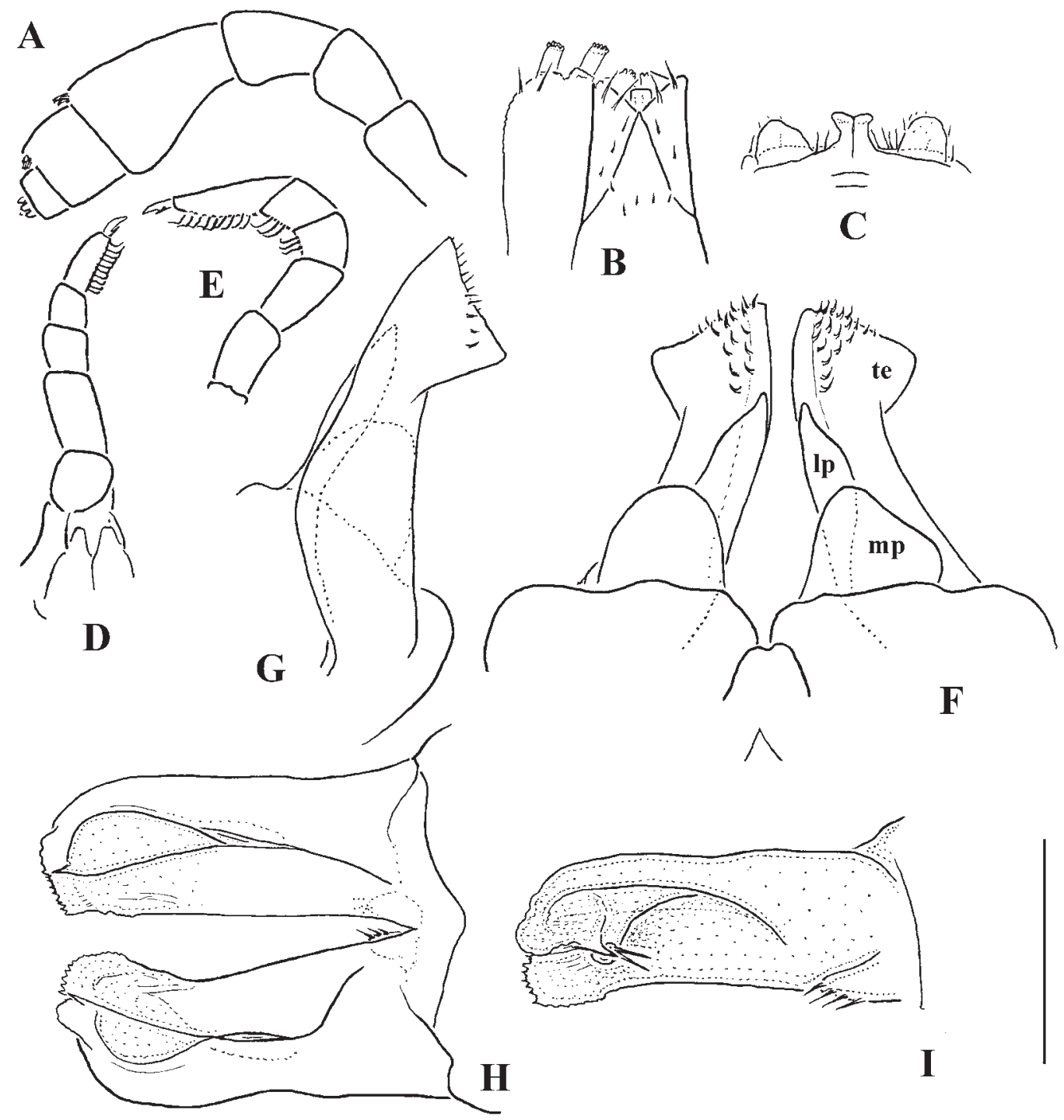

Fig. 23. Trachyjulus phylloides sp.n., OT paratype from Cave Tham Phra Khayang. A — antenna, lateral view; B - gnathochilarium, $\mathrm{C}$ - legs 1, front view; D - leg 2, caudal view; E - midbody leg, caudal view; F \& G - anterior gonopods, caudal and front views, respectively; $\mathrm{H}$ - posterior gonopods, front and caudal views, respectively. Scale bar: 0.4 (A-E) \& $0.2 \mathrm{~mm}$ (F-I).

Рис. 23. Trachyjulus phylloides sp.n. паратип О из пещеры Tham Phra Khayang. A — антенна, сбоку; В — гнатоъилярий, снизу; C - ноги 1, спереди; D - нога 2, сзади; Е — среднетуловищная нога, сзади; F и G - передние гоноподы, соответственно сзади и спереди; H - задние гоноподы, соответственно спереди и сзади. Масштаб: 0,4 (A-E), 0,2 мм (F-I).

or 6 striae to evident wrinkles. Segment 2 slightly, following metaterga fully, cristate, carinotaxy pattern of midbody segments typically $7-6 / 7-6+\mathrm{I} / \mathrm{i}+2 / 2+\mathrm{m} / \mathrm{m}$ (Fig. 19A-H). Postcollum constriction evident, but not particularly strong (Fig. 19A \& D). Midbody segments round in cross-section (Fig. 19H). Telson bare and smooth, paraprocts densely setose (Fig. 19C, F \& G).

Legs rather long and slender, a little longer than midbody height, devoid of modified setae (Figs 19AC, H, 20E). A rather long filament/spine at base of claw more than half the length of claw itself (Figs 19J, 20E). $\sigma^{7}$ legs 1-3 (Figs 20B-D) same as in numerous species of the Glyphiulus javanicus-group [Golovatch et al., 2007b, 2011c]; legs 1 with an unciform, rather short, doubled, central process flanked by similarly short, setose, sac-shaped, flattened telopodites.

Anterior gonopods (Figs 20F \& G) peculiar in lateral coxal process (lp) being slender, subequal in length to telopodite (te) and medial coxal process ( $\mathbf{m p}$ ), Ip clearly subtending te apex. Posterior gonopods (Figs 20H) shorter, ending in a shorter lateral lobe and a considerably higher, simple flagellum. 


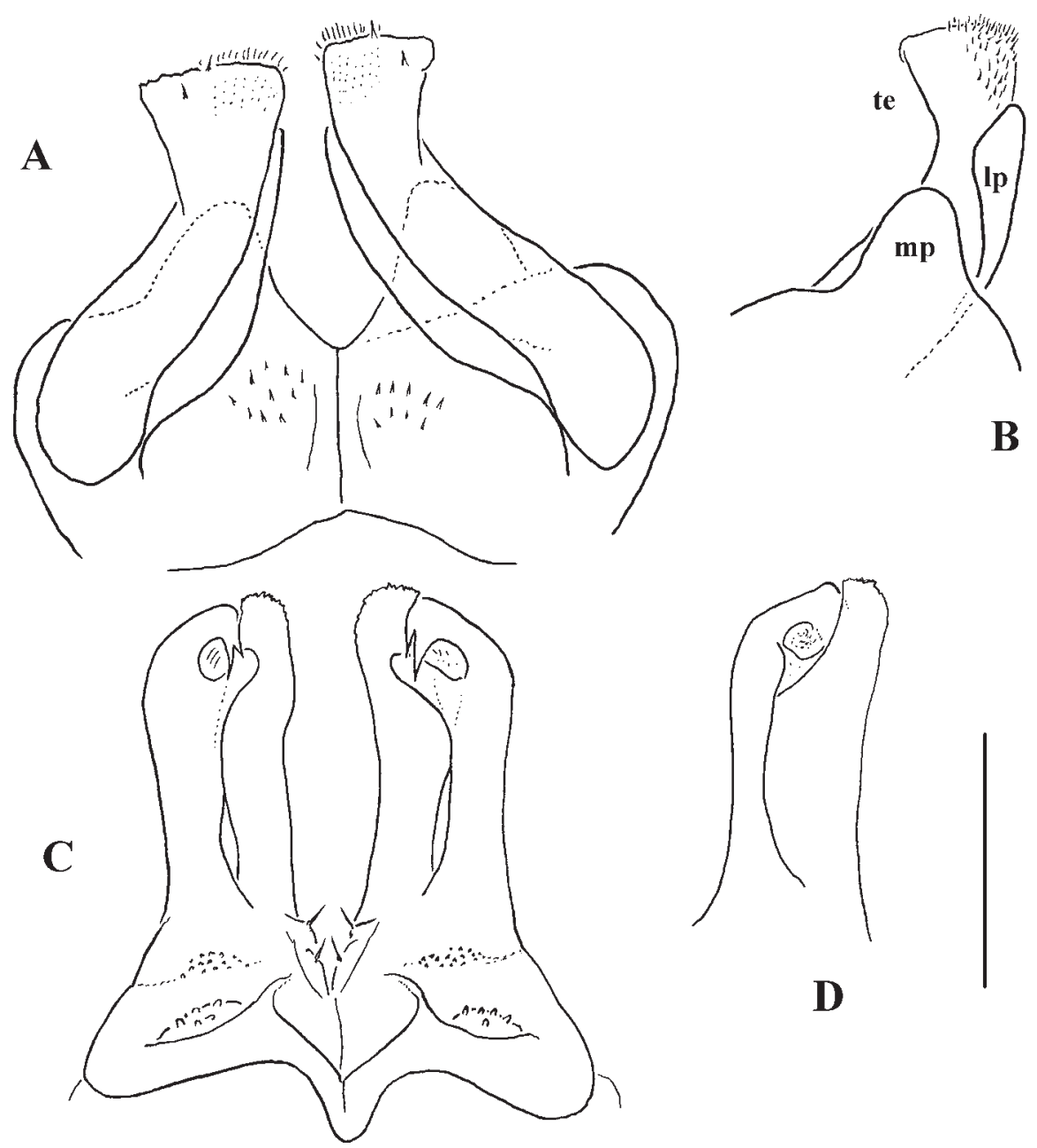

Fig. 24. Trachyjulus phylloides sp.n., $\mathrm{O}^{7}$ paratype from Cave Tham Kun Mung. A \& B - anterior gonopods, front and caudal views, respectively; C \& D - posterior gonopods, caudal and front views, respectively. Scale bar: $0.2 \mathrm{~mm}$.

Fig. 24. Trachyjulus phylloides sp.n., паратип О $0^{7}$ из пещеры Tham Kun Mung. A, В - передние гоноподы, соответственно спереди и сзади; C, D - задние гоноподы, соответственно сзади и спереди. Масштаб: 0,2 мм.

REMARKS. Because of its several troglomorphic traits, this species might well be a troglobite. So far as known, it occurs in a single cave (Map).

\section{Trachyjulus phylloides sp.n.}

Figs 21-24.

MATERIAL. Holotype $\sigma^{7}$ (MNHN GA 98), Thailand, Ranong Prov., Kraburi Distr., Cave Tham Phra Khayang, $18 \mathrm{~m}$ a.s.l., 9.12.2009, leg. S. Golovatch \& S. Panha (No. 31). Paratypes: 11

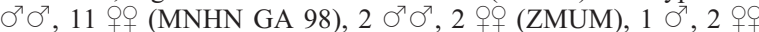
(ZMUC), $10^{7}, 1$ ( (MZCU), $10^{7}$ (SEM), same locality, together with holotype; 2 $\mathrm{O}^{\top} \mathrm{O}^{\top}, 2$ 우, 1 juv. (MNHN GA 98), 1 ( 9 (SEM), Chumphon Prov., Sawi, Cave Tham Kun Mung, bat guano, 28.07.1987, leg. L. Deharveng (THA-CPN-001)

NAME. To emphasize the peculiar, phylloid anterior gonopod telopodites, as well as most of the ventral setae on some $\sigma^{7}$ podomeres.

DIAGNOSIS. Differs from congeners by the peculiar, apically phylloid and strongly elongated telopodite of the anterior gonopods, coupled with the absence of a flagellum in bipartite posterior gonopods, and by $\sigma^{7}$ tibiae and tarsi beset with modified, apically phylloid setae.

DESCRIPTION. Length of adults ca $22-30 \mathrm{~mm}$ $\left(\sigma^{\top},{ }^{9}\right)$; width of midbody segments $0.9-1.1 \mathrm{~mm}\left(\sigma^{\top}\right.$, क). Adult body with $53-72 \mathrm{p}+3-2 \mathrm{a}+\mathrm{T}$ segments $\left(\sigma^{7},{ }^{\circ}\right)$. Holotype ca $30 \mathrm{~mm}$ long and $1.0 \mathrm{~mm}$ wide, with $72 \mathrm{p}+2 \mathrm{a}+\mathrm{T}$ segments. Coloration in adults rather variegate grey-brown to castaneous brown; pattern traceable in antennae, head, collum, venter and legs being yellow against brown metatergal crests and greyish interspaces. Ocelli brown to blackish.

3-4+3-4 ocelli in a single vertical row, obviously regardless of sex or size. Labrum with seven small teeth medially (Fig. 22A). Antennae rather short, slightly clavate (Figs 21A, 23A). Gnathochilarium (Figs 22A, 23B) rather oligotrichous, mentum single. Collum (Figs $21 \mathrm{~A} \&$ D) smooth, only near lateral edge with a few 
light, short, superficial striae. Segments 2 and 3 rather faintly, following metaterga fully, cristate, carinotaxy pattern of midbody segments typically $10-9 / 10-9+\mathrm{I} /$ $\mathrm{i}+2 / 2+\mathrm{m} / \mathrm{m}$ (Fig. 21). Postcollum constriction evident, but not particularly strong (Fig. 21A \& D). Midbody segments round in cross-section (Fig. 21I). Telson bare and smooth, paraprocts densely setose (Fig. 21C, $\mathrm{F} \& \mathrm{H})$.

Legs rather short and slender, evidently shorter than midbody height (Figs 21A-C, G, I, 22C, 23E). Ventral sides of $\sigma^{7}$ tarsi (mostly of tibiae and postfemora as well) beset with peculiar plumose to phylloid setae (Figs 22C, D, 23D \& E). A rather long filament/spine at base of claw a little to well over half the length of claw itself (Fig. 22E).

$\sigma^{7}$ legs 1-3 (Figs 22B, 23C \& D) same as in numerous species of the Glyphiulus javanicus-group [Golovatch et al., 2007b, 2011c]; legs 1 with an anchoriform, short, doubled, central process flanked by similarly short, setose, sac-shaped, flattened telopodites and then by much lower, lateral, setose knobs.

Anterior gonopods (Figs 22F-H, 23F, G, 24A \& B) peculiar in a slender lateral coxal process (lp) being about twice longer than a rounded, lobe-shaped, medial coxal process (mp), but in its turn much shorter than a large, apically setose, phylloid telopodite (te), lp not subtending te apex. Posterior gonopods (Figs 22F-H, $23 \mathrm{H}, \mathrm{I}, 24 \mathrm{C}-\mathrm{D})$ elongate, evidently bipartite, with a clear-cut fovea lying in distal third between both arms. delimited apically on front face of lateral arm by a couple of small spines; no trace of a flagellum.

REMARKS. Virtually nothing in the appearance of this species suggests its being a troglobite. So far as known, it only occurs in southern Thailand (Map).

ACKNOWLEDGEMENTS. We are most grateful to all persons who have entrusted us their valuable material for study. Sampling by Louis Deharveng and Anne Bedos (both MNHN) in Nusakambangan were supported by Fauna and Flora International - Indonesia; this couple kindly helped us also in preparing the map. Nesrine Akkari and Henrik Enghoff (both ZMUC) amiably helped us get clear pictures of $T$. nordquisti ambiguus from type material. The first author's stay in Paris in March and April 2012 was supported by the MNHN.

\section{References}

Attems C. 1903. Beiträge zur Myriopodenkunde // Zoologische Jahrbücher (Systematik). Bd.18. S.63-154.

Attems C. 1907. Javanische Myriopoden gesammelt von Direktor K. Kraepelin im Jahre 1903 // Mitteilungen aus dem Naturhistorischen Museum Hamburg. Bd.24. S.77-122.

Attems C. 1909. Die Myriopoden der Vega-Expedition // Arkiv för Zoologi. Bd.5. H.3. S.1-84.

Attems C. 1914. Die indo-australischen Myriopoden // Archiv für Naturgeschichte. Bd.80A. H.4. S.1-398.

Attems C. 1926. Myriopoda // Kükenthal W., Krumbach T. (Hrsg), Handbuch der Zoologie. Berlin \& Leipzig: W. de Gruyter \& Co. Bd.4. H.1. Progoneata, Chilopoda, Insecta. S.1-402

Attems C. 1927. Myriopoden von Ambon und anderen Inseln der Banda-See // Zoologische Mededeelingen, Rijks Museum van Natuurlijke Historie te Leiden. Deel 10. P.61-70.
Attems C. 1930. Myriopoden von Java, Sumatra und Bali // Archiv für Hydrobiologie. Suppl.-Bd.8. S.115-182.

Attems C. 1936. Diplopoda of India // Memoirs of the Indian Museum. Vol.11. P.133-323.

Attems C. 1938. Die von Dr C. Dawydoff in Französisch Indochina gesammelten Myriopoden // Mémoires du Muséum national d'Histoire naturelle. N.S. T.6. Fasc.2. P.187-353.

Carl J. 1911. Drei neue Diplopoden des Genfer Museums // Revue suisse de Zoologie. T.19. P.397-407.

Carl J. 1912. Die Diplopoden-Fauna von Celebes // Revue suisse de Zoologie. T.20. Fasc.4. P.73-206.

Carl J. 1922. Diplopoden aus Sumatra, Java, Malakka und Ceylon. Gesammelt von Herrn Ptof. Dr. v. Buttel-Reepen in den Jahren 1911-1912 // Zoologische Jahrbücher (Systematik). Bd.44. S.565-578.

Carl J. 1941. Diplopoden aus Südindien und Ceylon. 2. Teil: Nematophora und Juliformia // Ibid. T.48. P.569-714.

Chamberlin R.V. 1945. On some diplopods from the Indo-Australian Archipelago // American Museum Novitates. No.1282. P.1-43.

Enghoff H. 1993. Cape Verdean millipedes (Diplopoda // Tropical Zoology. Vol.6. P.207-216.

Golovatch S.I., Geoffroy J.-J., Mauriès J.-P., VandenSpiegel D. 2007a. Review of the millipede genus Glyphiulus Gervais, 1847, with descriptions of new species from Southeast Asia (Diplopoda, Spirostreptida, Cambalopsidae). Part 1. The granulatus-group // Zoosystema. Vol.29. Fasc.1. P.7-49.

Golovatch S.I., Geoffroy J.-J., Mauriès J.-P., VandenSpiegel D. 2007b. Review of the millipede genus Glyphiulus Gervais, 1847, with descriptions of new species from Southeast Asia (Diplopoda, Spirostreptida, Cambalopsidae). Part 2. The javanicus-group // Zoosystema. Vol.29. Fasc.3. P.417-456.

Golovatch S.I., Geoffroy J.-J., Mauriès J.-P., VandenSpiegel D. 2009. Review of the millipede genus Plusioglyphiulus Silvestri, 1923, with descriptions of new species from Southeast Asia (Diplopoda, Spirostreptida, Cambalopsidae) // Zoosystema. Vol.31. Fasc.1. P.71-116.

Golovatch S.I., Geoffroy J.-J., Mauriès J.-P., VandenSpiegel D. 2011a. The millipede genus Plusioglyphiulus Silvestri, 1923 in Thailand (Diplopoda, Spirostreptida, Cambalopsidae) // Zootaxa. No.2840. P.1-63.

Golovatch S.I., Geoffroy J.-J., Mauriès J.-P., VandenSpiegel D. 2011b. New species of the millipede genus Glyphiulus Gervais, 1847 from the granulatus-group (Diplopoda: Spirostreptida: Cambalopsidae)// Arthropoda Selecta. Vol.20. No.2. P.65-114.

Golovatch S.I., Geoffroy J.-J., Mauriès J.-P., VandenSpiegel D. 2011c. New species of the millipede genus Glyphiulus Gervais, 1847 from the javanicus-group (Diplopoda: Spirostreptida: Cambalopsidae) // Arthropoda Selecta. Vol.20. No.3. P.149-165.

Golovatch S.I., Geoffroy J.-J., Mauriès J.-P., VandenSpiegel D. 2011d. Two new species of the millipede genus Hypocambala Silvestri, 1895 from China and Vietnam (Diplopoda: Spirostreptida: Cambalopsidae) // Arthropoda Selecta. Vol.20. No.3. P.167-174.

Hoffman R.L. 1977. Diplopoda from Malayan caves collected by M. Pierre Strinati // Revue suisse de Zoologie. T.84. Fasc.3. P.699-719.

Jeekel C.A.W. 1963. Diplopoda of Guiana (1-5) // Studies on the Fauna of Suriname and other Guyanas. Vol.4. No.11. P. $1-157$.

Jeekel C.A.W. 2004. A bibliographic catalogue of the "Cambaloidea" (Diplopoda, Spirostreptida) // Myriapod Memoranda. Vol.7. P.43-109.

Mauriès J.-P. 1970. Examen des types des genres Cambalomorpha et Cambalopsis Pocock, 1895. Essai de classification des Glyphiulinae Verhoeff, 1936 (Diplopoda, Cambalidea) // Bulletin du Muséum national d'Histoire naturelle. 2e sér. T.42. Fasc.3. P.509-519

Mauriès J.-P. 1981. Craspedosomida, Stemmiulida et Cambalida (Myriapoda: Diplopoda) de Sri Lanka (Ceylan) // Entomologica Scandinavica. Suppl.11. P.33-62. 
Mauriès J.-P. 1983. Cambalides nouveaux et peu connus d'Asie, d'Amérique et d'Océanie. I. Cambalidae et Cambalopsidae (Myriapoda, Diplopoda) // Bulletin du Muséum national d'Histoire naturelle, 4e sér., sect. A. T.5. Fasc.1. P.247-276.

Peters W. 1864. Übersicht der im Königl. Zoologischen Museum befindlichen Myriopoden aus der Familie Polydesmi, so wie Beschreibungen einer neuen Gattung, Trachyjulus, der Juli und neuer Arten der Gattung Siphonophora // Monatsberichte der Königlich Preußischen Akademie der Wissenschaften zu Berlin. Bd.1864. S.529-551.

Pocock R.I. 1892. Report upon two collections of Myriopoda sent from Ceylon by Mr. E.E. Green, and from various parts of southern India by Mr. Edgar Thurston, of the Government Central Museum, Madras // Journal of the Bombay Natural History Society. Vol.7. P.131-174.

Pocock R.I. 1893. Report upon the Julidae, Chordeumidae and Polyzonidae collected by Sig. L. Fea and Mr. E. W. Oates // Annali del Museo Civico di Storia Naturale di Genova. T.33. P.386-406.

Pocock R.I. 1894. Chilopoda, Symphyla and Diplopoda from the Malay Archipelago // Weber M. (Hrsg.). Zoologische Ergebnisse einer Reise in Niederländisch Ost-Indien. Bd.3. S.307404.

Pocock R.I. 1895. Report upon the Chilopoda and Diplopoda obtained by P. W. Basset-Smith, Esq., Surgeon R. N., during the cruise in the Chinese seas of H.M.S. "Penguin", Commander
W. U. Moore Commanding // Annals and Magazine of Natural History. Ser. 6. Vol.15. P.346-372.

Schubart O. 1946. "Cambalopsis nordquisti" Attems da Ásia oriental, habitante do Distrito Federal do Brasil (Diplopoda, Cambalopsidae) // Revista Brasileira de Biologia. T.6. No.3. P.395-406.

Silvestri F. 1895. I chilopodi ed i diplopodi di Sumatra e delle isole Nias, Engano e Mentavei // Annali del Museo Civico di Storia Naturale di Genova. Ser. 2. T.14(34). P.707-760.

Silvestri F. 1923. Descriptions of some Indian and Malayan Myriapoda Cambaloidea // Records of the Indian Museum. Vol.25. Pt.2. P.181-193.

Silvestri F. 1924. Myriapoda from the Siju Cave, Garo Hills, Assam // Records of the Indian Museum. Vol.26. P.71-79.

Verhoeff K.W. 1936. Zur Kenntnis der Glyphiuliden (Cambaloidea). 143. Diplopoden-Aufsatz // Zoologischer Anzeiger. Bd.113. H.3/4. S.49-62.

Wang Y.H.-M. 1967. Serica IV: Millipedes and centipedes from Bukit Timah and from Cameron Highlands, Malaysia // Quarterly Journal of the Taiwan Museum. Vol.20. P.393-398.

Wang Y.H.-M., Tang M.C. 1965. Serica IR: The millipedes of Malay Archipelago and South Sea Islands: Singapore, Sarawak and Sumatra // Ibid. Vol. 18. P.399-441.

Responsible editor K.G. Mikhailov 